\title{
Statistical Studies of Supernova Environments
}

\author{
Joseph P. Anderson ${ }^{1,5}$, Phil A. James ${ }^{2}$, Stacey M. Habergham² ${ }^{2}$ Lluís Galbany ${ }^{3,4}$ \\ and Hanindyo Kuncarayakti ${ }^{3,4}$ \\ ${ }^{1}$ European Southern Observatory, Alonso de Cordova 3107, Vitacura, Casilla 19001, Santiago, Chile \\ ${ }_{2}^{2}$ Astrophysics Research Institute, Liverpool John Moores University, IC2, Liverpool Science Park, 146 Brownlow Hill, Liverpool L3 5RF, UK \\ ${ }^{3}$ Millennium Institute of Astrophysics, Santiago, Chile \\ ${ }^{4}$ Departamento de Astronomía, Universidad de Chile, Santiago, Casilla 36-D, Chile \\ 5Email: janderso@eso.org
}

(ReCeIved December 12, 2014; ACCEPTED April 15, 2015)

\begin{abstract}
Mapping the diversity of SNe to progenitor properties is key to our understanding of stellar evolution and explosive stellar death. Investigations of the immediate environments of SNe allow statistical constraints to be made on progenitor properties such as mass and metallicity. Here, we review the progress that has been made in this field. Pixel statistics using tracers of e.g. star formation within galaxies show intriguing differences in the explosion sites of, in particular SNe types II and Ibc (SNe II and SNe Ibc respectively), suggesting statistical differences in population ages. Of particular interest is that $\mathrm{SNe}$ Ic are significantly more associated with host galaxy $\mathrm{H} \alpha$ emission than $\mathrm{SNe} \mathrm{Ib}$, implying shorter lifetimes for the former. In addition, such studies have shown (unexpectedly) that the interacting SNe IIn do not explode in regions containing the most massive stars, which suggests that at least a significant fraction of their progenitors arise from the lower end of the core-collapse SN mass range. Host H II region spectroscopy has been obtained for a significant number of core-collapse events, however definitive conclusions on differences between distinct SN types have to-date been elusive. Single stellar evolution models predict that the relative fraction of SNe Ibc to SNe II should increase with increasing metallicity, due to the dependence of mass-loss rates on progenitor metallicity. We present a meta-analysis of all current host $\mathrm{H}$ II region oxygen abundances for CC SNe. It is concluded that the SN II to SN Ibc ratio shows little variation with oxygen abundance, with only a suggestion that the ratio increases in the lowest bin. Radial distributions of different SNe are discussed, where a central excess of SNe Ibc has been observed within disturbed galaxy systems, which is difficult to ascribe to metallicity or selection effects. Environment studies are also being undertaken for SNe Ia, where constraints can be made on the shortest delay times of progenitor systems. It is shown that 'redder' SNe Ia are more often found within star-forming regions. Environment studies are evolving to enable studies at higher spatial resolutions than previously possible, while in addition the advent of wide-field integral field unit instruments allows galaxy-wide spectral analyses which will provide fruitful results to this field. Some example contemporary results are shown in that direction.
\end{abstract}

Keywords: galaxies: star formation - galaxies: statistics - HII regions - supernovae: general

\section{INTRODUCTION}

Supernovae ( $\mathrm{SNe}$ ) are the explosive deaths of stars. The light released is such that the explosion can for a brief time of weeks to months, be close in luminosity to the total light produced by its host galaxy, i.e. billions of individual stars. These extreme luminosities and their associated energies mean that $\mathrm{SNe}$ are not only interesting to understand in their own right, but are also important objects which heavily influence their immediate environments and the evolution of those regions. In addition, $\mathrm{SNe}$ can also be used as probes to aid in our understanding of many other astrophysical questions.

There now exists a large diversity of SN type classifications, with new distinct events being added to the traditional observational classes. SNe were originally classified into types I and II, due to the absence in the former and the presence in the latter, of hydrogen in their spectra (Minkowski 1941). Later, the type I class was further sub-divided as it became clear that further distinct types exist. $\mathrm{SNe} \mathrm{Ib}$ show helium in their spectra, while SNe Ic do not, and both lack the strong silicon absorption characteristic of SNe Ia (see Filippenko 1997 for a review of SN spectral classifications) ${ }^{1}$. $\mathrm{SNe}$ II can also be further split by properties of their lightcurve and spectra. SNe IIP are observed to have a 'plateau' in their light-curves, while the faster declining 'linear' events

\footnotetext{
${ }^{1}$ Throughout the manuscript, we will refer to the combined sample of SNe Ib,
} $\mathrm{SNe}$ Ic, and those classified as 'Ib/c', as SNe Ibc. 
are classified as SNe IIL (Barbon, Ciatti, \& Rosino 1979, although see Arcavi et al. 2012, Anderson et al. 2014b and Faran et al. 2014a for discussion on SN II typing). Two further type II spectroscopic classifications exist. SNe IIn are those events which show narrow emission features in their spectra (Schlegel 1990), indicating that their ejecta are interacting with slow moving circumstellar material (CSM, Chugai \& Danziger 1994). At early times $\mathrm{SNe}$ IIb show features similar to normal SNe II, but later hydrogen features disappear, and they appear more similar to SNe Ib (Filippenko, Matheson, \& Ho 1993). In addition, there also exist a subset of the SN Ic class which show broad spectral features implying high ejecta velocities. These are known as 'broad-line (BL) SNe Ic' (or SNe Ic BL), and a further subset of SNe Ic BL have been linked to long-duration Gamma Ray Bursts (LGRBs, see e.g. Galama et al. 1998; Hjorth et al. 2003).

From a theoretical viewpoint, historically SN progenitors have been separated into two classes. SNe Ia are presumed to arise from the thermonuclear disruption of a white dwarf (WD) or WD system, while core-collapse (CC) SNe (i.e. types II and Ibc) are thought to be the result of the $\mathrm{CC}$ of massive stars. This separation is given further credence by the fact that $\mathrm{CC}$ SNe are only observed in star-forming latetype galaxies, while SNe Ia are observed in all galaxy types (including ellipticals, van den Bergh, Li, \& Filippenko 2005). Given that elliptical galaxies are dominated by evolved stellar populations, this effectively constrains the $\mathrm{SNe}$ occurring within those galaxies to arise from similarly evolved progenitors. A WD progenitor can easily accommodate this constraint. Meanwhile, the fact that $\mathrm{CC} \mathrm{SNe}$ are exclusively found within galaxies containing significant star formation (SF), is consistent with their progenitors being the collapse of the cores of massive stars. However, outside of these very broad progenitor constraints of young ( $\mathrm{CC} \mathrm{SNe}$ ) and old (SNe Ia) parent populations, gaining further information on the specific progenitor parameter space which defines the observed transient diversity has been relatively slow going.

In recent years, the traditional classification scheme has been further complicated by the addition of peculiar types such as SNe Iax (see e.g. Foley et al. 2013 and references therein) and 'Ca-rich' events (e.g. Perets et al. 2010). The features in these distinct transients give clues as to the initial progenitor and pre-SN characteristics giving rise to the observed SN diversity. Indeed, there is debate as to how these events can be understood in terms of the classic supposedly fundamental theoretical classes.

It is generally accepted that CC SN diversity can be explained through the amount of the progenitor star's envelope retained at the epoch of explosion. This then defines the dominant spectral features, and the shape of the light-curve. The main question then becomes: which progenitor property defines the amount of mass lost by the star or stellar system before explosion? Initial progenitor mass, metallicity, and rotation, together with the presence and influence of a binary companion all affect this degree of mass loss. The epoch and rate of that mass loss can also heavily influence the ob- served SN, where in e.g. SNe IIn, observations suggest that mass-loss occurred close to the epoch of explosion, causing the large dispersion in properties observed in this class (see e.g. Kiewe et al. 2012; Taddia et al. 2013b). Deciphering which changes in progenitor properties link to changes in $\mathrm{SN}$ features is then a key question. Studying the immediate environments where $\mathrm{SNe}$ explode can help elucidate these issues.

The most direct way to constrain SN progenitors is to observe the progenitor star before explosion. This has been possible in a small number of cases, and for SNe IIP these studies have constrained their progenitors to be red supergiant (RSG) stars with initial masses between 8 and $16 \mathrm{M}_{\odot}$ (see Smartt 2009 for a review). This technique requires that pre-SN images of sufficient depth at SN positions are available. One can then use photometry of the star coincident with explosion locations to derive a luminosity and convert this to a progenitor mass using the predictions of stellar evolution codes. While this technique is highly enlightening with respect to individual $\mathrm{SN}$ events, its use in a statistical sense is limited by (a) the lack of explosions close enough where one can detect/resolve individual stars, and (b) the need for sufficiently deep pre-explosion images. Hence, to build up statistics for the different SN types through this avenue will take several more decades. At the other end of the progenitor constraint spectrum is the analysis of global host galaxy properties. Indeed as mentioned above, the separation of SNe into $\mathrm{CC}$ and thermonuclear events owes much to the appearance of the latter in early-type elliptical galaxies.

More recent studies have investigated how e.g. the SN Ibc to SN II ratio changes with host galaxy mass/luminosity (Prantzos \& Boissier 2003; Boissier \& Prantzos 2009; Arcavi et al. 2010), metallicity (Prieto, Stanek, \& Beacom 2008a), host morphology (Hakobyan et al. 2014), and the multiplicity of SNe within different galaxies (Anderson \& Soto 2013). These studies show intriguing differences, and allow for statistically significant analyses. However, especially in latetype spiral galaxies, there are multiple stellar populations of distinct ages, metallicities, and possible SF scenarios. This therefore makes drawing definitive conclusions on progenitor properties complicated. An interesting avenue for investigation which overcomes some of the limitations of the above is to analyse the stellar populations at the locations of $\mathrm{SNe}$ within host galaxies. In this direction, one can still obtain significant statistics (as will be shown below), while analysing stellar populations that are more representative of SN progenitors than global host investigations.

In this review, we will summarise the progress that has been made in the field of SN 'environments'. The review is outlined as follows. First, we continue this introduction with a short history of environmental studies, discussing the important constraints that have been made on progenitors, and bringing the reader up-to-date with the previous decade. The introduction finishes with a description of the definition of what we mean when analysing SN 'environments'. We then outline the different statistical techniques that have recently 
been used in this field and this is followed by a discussion of the important results derived from environment analysis in Section 3. In Section 4, we further discuss the implications of these results, and the various biases and complications in their interpretation. In Section 5, the current and future status of this field is discussed, and finally in Section 6 we conclude with an overall summary.

\subsection{A short history of supernova environment studies}

The first environmental evidence used to constrain the physical properties of $\mathrm{SNe}$ arose due to the observation that type I SNe (at that stage no separation was made between type Ia and type Ibc) were found to explode in early-type elliptical galaxies, while type II events were found exclusively within star-forming galaxies (see e.g. Reaves 1953 for an early example, confirmed by many subsequent studies, e.g. van den Bergh et al. 2005, Hakobyan et al. 2014). However, even further back in time Baade \& Zwicky (1934) made the observation that ' ... super-novae occur not only in the blurred central parts of nebulae but also in the spiral arms...'. This was reference to the spatial distribution of novae and $\mathrm{SNe}$ within their host galaxies and hence environmental information was already used through those early investigations to note differences in the properties between these two events. Johnson \& MacLeod (1963) concluded that all SN types were concentrated in the disks of galaxies. Progenitor constraints from host galaxies were given further strength through research investigating the correlation of SNe with spiral arms (thought to trace SF) within galaxies. Maza \& van den Bergh (1976) demonstrated that SNe II were much more concentrated within spiral arms than SNe I (also see Huang 1987) ${ }^{2}$, again implying shorter lived progenitors for the former (McMillan \& Ciardullo 1996 later showed that this difference indeed exists between pure samples of SNe II and SNe Ia). While spiral arms trace the general waves of SF within galaxies, on a more local scale high mass ongoing SF is traced by $\mathrm{H}$ II regions. Hence, the next logical step was pursued and analyses were carried out investigating the association of different $\mathrm{SN}$ types with these regions by van Dyk (1992), Bartunov, Tsvetkov, \& Filimonova (1994) and van Dyk, Hamuy, \& Filippenko (1996). These authors estimated the different degrees of association of $\mathrm{SN}$ types to $\mathrm{H}$ II regions through measuring the distance to the nearest region from each explosion site. At this epoch, SNe I had now been separated into SNe Ia and $\mathrm{SNe} \mathrm{Ibc}$, and the main conclusion from these studies was that any significant difference between the correlation of SNe II and SNe Ibc (both arising from massive stars) and $\mathrm{H}$ II regions was not observed (which, with larger samples and a distinct analysis technique we show to be incorrect below).

\footnotetext{
${ }^{2}$ Note, while in these studies there was probably a mix of SNe Ia and SNe Ibc, given their relative rates, most of the sample was probably dominated by $\mathrm{SNe}$ Ia, hence the significant difference in correlation.
}

Another analysis technique used historically, is to investigate the radial distribution of different $\mathrm{SN}$ types, and compare these to our understanding of radial trends of e.g. metallicity in spiral galaxies. Bartunov, Makarova, \& Tsvetkov (1992) concluded there were no statistical differences in the radial distributions of different $\mathrm{SN}$ types. However, later investigations by van den Bergh (1997), Wang, Höflich, \& Wheeler (1997) and Tsvetkov, Pavlyuk, \& Bartunov (2004) showed that SNe Ibc are generally more centrally concentrated within galaxies than SNe II, while SNe Ia appeared to show a deficit of events within central regions. The central bias of SNe Ibc with respect to other SN types has historically been interpreted as a metallicity difference as the central parts of spiral galaxies are generally host to higher metallicity stellar populations (due to metallicity gradients within galaxies, see Henry \& Worthey 1999 for a review). However, as we will show below, this interpretation is complicated when one delves deeper into specific host galaxy properties.

In the above, the reader has been brought up to date to the sparse 'environment' studies initially undertaken during the 20th century. The rest of this review will go into detail on the 'environment' studies of the last decade, where this field has substantially matured. First, for the sake of the rest of the article, we will define what we mean by 'environment' in the context of the current review.

\subsection{A definition of 'environments'}

In this paper, we define $\mathrm{SN}$ environments studies as follows:

1. Using observations when the light from the $\mathrm{SN}$ is not contaminating that of the parent stellar population, i.e. either before the SN has exploded, or once it has faded below the luminosity of the environment light. This constraint is important in order to analyse the parent stellar population light uncontaminated by SN emission.

2. Use of observations of unresolved (into individual stars) stellar populations. This then rules out analyses of resolved stellar populations in very nearby galaxies. While results from such studies can be very revealing (and indeed will be discussed later), this constraint focusses the discussion on unresolved populations.

3. Analyses where one separates host galaxies into distinct regions. This distinguishes environmental studies from global host work. While this factor is somewhat due to the researcher's own choice, it is also affected by distance resolution effects. As one goes to larger distances, it becomes more difficult to resolve individual galaxies into separate detector pixels.

To obtain progenitor constraints on $\mathrm{SNe}$, one then assumes that stellar population properties are representative of those of progenitor stars/stellar systems. Given the short lifetimes of $\mathrm{CC} \mathrm{SNe} \mathrm{(at} \mathrm{most} \mathrm{several} \mathrm{tens} \mathrm{of} \mathrm{Myrs,} \mathrm{although} \mathrm{note}$ 
the possibility of longer lived CC SN progenitors produced in binary systems), this assumption is most likely valid. In the case of SNe Ia, their longer delay times before explosion make interpretations more problematic. There are also issues with chance alignments of $\mathrm{SNe}$ onto stellar populations which are not associated with those of the progenitors. These issues and others will be further discussed in Section 4.

\section{STATISTICAL TECHNIQUES}

Environmental analyses have been undertaken through both photometric and spectroscopic avenues. Photometric analyses have focussed on the flux at the particular explosion sites through different broad- or narrow-band filters, or multiple filters tracing environment colours (e.g. Kelly \& Kirshner 2012). These observations can be linked to particular properties of stellar populations, predominantly age, but also line-of-sight extinction, and metallicity (through stellar population modelling). Analyses of SN radial distributions, as normalised to stellar population distributions throughout galaxies have also been undertaken. Spectroscopic analyses have generally concentrated on metallicity derivations through emission line ratio diagnostics, however equivalent width (EW) measurements (see e.g. Kuncarayakti et al. 2013a and further discussion below), have also been used.

The general methods of these studies will now be outlined; however, the reader is encouraged to delve deeper into individual references for a complete description of these techniques, their possible biases and error estimations.

\subsection{Host galaxy pixel statistics}

SN environment studies started in earnest with the introduction of host galaxy 'pixel statistics'. In 2006, two papers independently developed very similar techniques with the aim of analysing where within the distribution of pixel counts of particular galaxies the SN environment falls. James \& Anderson (2006) investigated the association of different SN types with host galaxy ongoing SF, through narrow-band $\mathrm{H} \alpha$ observations, while Fruchter et al. (2006) analysed the association of LGRBs, to host galaxy $g^{\prime}$-band light as compared to CC SNe. The majority of the below discussion will focus on the analysis using the James \& Anderson method, that will now be described in more detail. However, the Fruchter et al. method is very similar (and has also been used subsequently by e.g. Kelly, Kirshner, \& Pahre 2008).

The description to provide pixel statistics (dubbed 'NCR' values in Anderson \& James 2008) is outlined below with respect to $\mathrm{H} \alpha$ imaging. However, this description is valid for analysis of host galaxy observations in any waveband (and indeed could be applied to spectral observations as we will outline in Section 5). To begin, continuum-subtracted $\mathrm{H} \alpha$ images are trimmed to remove sky regions outside the galaxy. Then, pixel counts within the resulting image are ordered in terms of increasing count. From this, the cumulative distribution is formed. This cumulative distribution is then normalised by dividing by the total count of all pixels. This then leaves negative values, followed by NCR values between 0 and 1. Negative NCR values, the result of noise in the sky, are then set to 0 . Thus, this statistic is made such that values of 0 are equal to sky or zero flux, while a value of 1 means that the pixel contains the highest flux of the whole galaxy. The final NCR value can thus be understood as the fraction of $\mathrm{H} \alpha$ flux arising from pixels with lower counts than the SN-containing pixel. One can then proceed to measure NCR values for different $\mathrm{SNe}$ within different galaxies, and build statistical samples to be compared. If a distribution accurately follows the (in this example case) $\mathrm{H} \alpha$ emission, then one expects the distribution to be flat with a mean NCR of 0.5 . This would imply that in a population that accurately traces host galaxy $\mathrm{H} \alpha$ emission, an equal number of $\mathrm{SNe}$ have NCR values in each bin of NCR (for more details and discussion see Anderson et al. 2012).

Using pixel statistics, one can use differences in the association of SN types to tracers of different stellar populations to constrain progenitor properties. In the above example of $\mathrm{H} \alpha$ emission, this then gives differences in associations to very young stellar populations, and hence can be used to probe relative age differences between progenitors, as will be shown below.

The spatial resolutions probed by pixel statistics depend on the distance of the host galaxies observed, together with the pixel scale of the instrument used and the image quality of those observations. In the case of $\mathrm{H} \alpha \mathrm{NCR}$ statistics, which will dominate the discussion below, the median physical sizes probed are on the order of $300 \mathrm{pc}$ (see Anderson et al. 2012, 2015 for more detailed estimations). Hence, in general these statistics do not probe individual $\mathrm{H}$ II regions, but larger starforming regions within galaxies.

\subsection{Radial distributions}

A more generalised environmental parameter than that of the above pixel statistics, is the radial position within hosts where $\mathrm{SNe}$ are found. Within galaxies the properties of stellar populations can change significantly with radial position. Metallicity gradients can exist (see e.g. Henry \& Worthey 1999), in that the central parts of galaxies are generally more metal rich than outer regions. There also exist distinct populations in stellar bulges and bars within the centres of galaxies. Galaxy interactions and mergers can also significantly affect the SF processes within galaxies. Hence, one can analyse differences in the radial distributions of $\mathrm{SNe}$ and try to understand these in the context of the different stellar populations found at different galactocentric radii.

The first studies to investigate the radial distributions of $\mathrm{SNe}$ generally normalised galactocentric distances to e.g. $R_{25}$ (radius of galaxy to the 25th mag. isophote) or worked with absolute distances. One issue with this type of normalisation is that it does not take into account the actual radial 
distributions of different stellar populations. James \& Anderson (2006) analysed the radial distributions of SNe with respect to those of the light as seen through distinct filters. This analysis measures the ellipse with the same parameters as that of their host galaxy that just includes each SN. One then measures the flux contained within this ellipse, and normalises to the flux contained within an ellipse out to distances where the galaxy flux is consistent with sky values. One again obtains values between 0 and 1 , where a value of 0 would mean that a particular SN occurs at the central peak of emission, while a value of 1 means that the $\mathrm{SN}$ occurs out at distances where no significant galaxy flux is measured. Building radial distributions of different $\mathrm{SN}$ types one can then analyse differences and try to tie these to differences in progenitor properties. In the below discussion, we concentrate on SN radial distributions with respect to continuum $R$-band light, which is referred to as $F r_{R}$. For a full discussion of these analysis methods, see James \& Anderson (2006) and Anderson \& James (2009). There are several selection effects which may be at play in such an analysis, mostly due to missing $\mathrm{SNe}$ in the central parts of host galaxies due to the high surface brightness background. However, as has been shown in Habergham, James, \& Anderson (2012), there is no significant evidence that these issues will affect one SN type preferentially over the other, especially in the case of differences between SNe II and SNe Ibc. In Section 4.5, we discuss selection effects in general with respect to environment analyses.

\subsection{H II region metallicities}

CC SN progenitor metallicity is predicted to play a significant role in the determination of SN type, especially in the single star progenitor scenario (see predictions from e.g. Heger et al. 2003). In extra-galactic studies, the simplest way to measure stellar population metallicity is through observing the ratio of strong emission lines produced through the ionisation of ISM material by massive stars. The metallicity of choice is that of oxygen, as this is the most abundant metal found in $\mathrm{H}$ II regions and shows strong emission lines in $\mathrm{H}$ II region spectra. In addition, ISM oxygen abundances show good correlation with stellar metallicities of massive stars (see e.g. Simón-Díaz \& Stasińska 2011). While there now exist a plethora of oxygen abundance diagnostics, there is significant disagreement between different diagnostics, meaning that constraints on $\mathrm{H}$ II region metallicities in absolute terms are somewhat problematic. A detailed discussion of these issues, together with suggestions of which diagnostic to use for specific cases is given in Kewley \& Ellison (2008). While oxygen abundance measurements between diagnostics can often show large offsets, relative metallicities within a given diagnostic are thought to be much more reliable. Hence, one can investigate $\mathrm{H}$ II region metallicities between different $\mathrm{SN}$ types, using a specific diagnostic.

While the ISM metallicity presents the gas abundance at the epoch of observation, given the relatively short lifetimes of CC SNe (at most several 10s of Myrs) one can assume that the $\mathrm{SN}$ host $\mathrm{H}$ II region metallicity is a reasonable tracer of progenitor metallicity. One issue is that it is common that at exact explosion sites emission lines are relatively weak, meaning one has to observe the nearest emission line region. Indeed, this is shown in Section 3.1, where particularly for $\mathrm{SNe}$ II (but also for many $\mathrm{SNe} \mathrm{Ib}$, and a small number of SNe Ic), CC SNe are found to explode within environments with relatively low $\mathrm{H} \alpha$ flux. While it is not clear that this should bring any systematic bias to CC SN metallicity measurements, it is important to note the complications in direct progenitor metallicity constraints from environments. Some studies have attempted to negate this problem by only analysing metallicities which have been extracted at exact explosion sites. However, if there is some progenitor effect which determines whether an SN is less or more likely to explode within a bright $\mathrm{H}$ II region (which we argue there is), then one may be simply replacing one bias with another. Hence, in our analysis below we include all 'environment' metallicity measurements. We note that a relatively recent review of metallicity measurements of $\mathrm{CC} \mathrm{SNe}$ and those SNe accompanying LGRBs, was also presented in Modjaz (2011).

There have now been multiple studies of CC SN host $\mathrm{H}$ II region metallicities (Modjaz et al. 2008; Anderson et al. 2010; Modjaz et al. 2011; Leloudas et al. 2011; Modjaz 2011; Sanders et al. 2012; Kuncarayakti et al. 2013a, 2013b; Stoll et al. 2013; Taddia et al. 2013a). However, overall conclusions on progenitor metallicities implied from these results are still not definitive (more details from these studies will be discussed below). In this review, we present a meta-analysis, combining all of the above measurements to try to further probe whether environment metallicity differences between SN types indeed exist. The most common metallicity calibration used is that from Pettini \& Pagel (2004), and hence we take those values for our analysis. Apart from being the commonly used diagnostic (and hence published abundance values being readily available), the PP04 metallicity scale also has the advantage that it is derived using emission lines very close in wavelength: $\mathrm{H} \alpha$ together with [N II], and $\mathrm{H} \beta$ together with [O III]. This means that uncertainties in extinction corrections and/or relative spectral flux calibration are unimportant. The PP04 scale has two metallicity indicators: $\mathrm{N} 2$, using only $\mathrm{H} \alpha$ and [ $\mathrm{N}$ II] and $\mathrm{O} 3 \mathrm{~N} 2$, which additionally uses $\mathrm{H} \beta$ and [O II] line strengths. In a significant number of cases publications only list $\mathrm{N} 2$ or $\mathrm{O} 3 \mathrm{~N} 2$ values (the former because in many cases one only detects $\mathrm{H} \alpha$ and [N II]). As these two diagnostics are on the same scale (which is tied to the ISM electron temperature), we use a combination of both values, preferring $\mathrm{O} 3 \mathrm{~N} 2$, but using $\mathrm{N} 2$ when the former is not available.

In the rest of this review, we will concentrate on analysis and subsequent discussion of the results obtained through the above methods. However, there are specific cases which are not covered by the above and these will be introduced and discussed separately. 


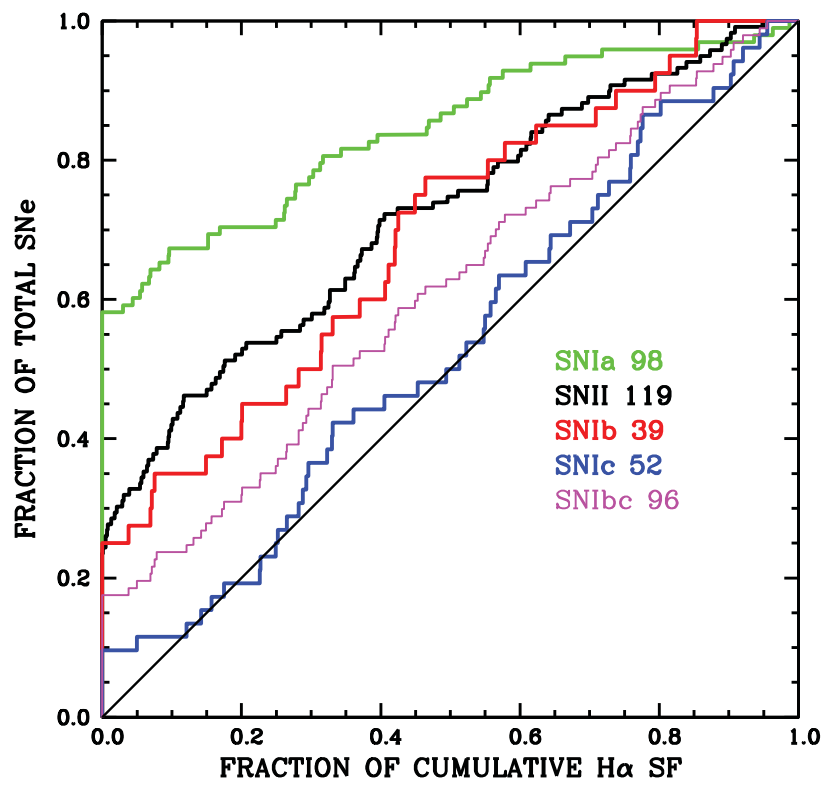

Figure 1. Cumulative $\mathrm{H} \alpha$ NCR distributions of the main SN types: SNe Ia, $\mathrm{SNe} \mathrm{II}, \mathrm{SNe} \mathrm{Ib}$, and SNe Ic (the combined SN Ibc population is also shown). The straight black diagonal line represents a hypothetical distribution, infinite in size, which accurately traces (in this case) host galaxy on-going SF as traced by $\mathrm{H} \alpha$ line emission. This plot is a reproduction of Figure 2 from Anderson et al. (2012), but here we remove the SNe IIb and SNe IIn from the SN II sample, to be consistent in our sample analysis throughout the review.

\section{SUPERNOVA ENVIRONMENTS}

Results will first be summarised concentrating on the main CC SN types of SNe II and SNe Ibc, through pixel statistics, radial distributions, and host $\mathrm{H}$ II region spectral analyses. This is followed by some discussion of the environment studies which fall outside of these analysis methods. Then SNe Ia environments will be discussed. We will make some comments on specific rarer SN sub-types, where environmental analyses aid in simply deciding between a CC and thermonuclear origin. Finally, we will present some results where environmental properties are linked to specific SN properties, beyond classical spectral/light-curve classifications.

\subsection{Pixel statistics}

In Figure 1, the $\mathrm{H} \alpha$ NCR pixel statistics cumulative distributions of: $\mathrm{SNe} \mathrm{Ia}, \mathrm{SNe}$ II, $\mathrm{SNe} \mathrm{Ib}$, and $\mathrm{SNe}$ Ic are presented (taken from Anderson et al. 2012). These plots (which will be repeated in various forms below) can be understood in the following way. As outlined above, if a population accurately traces the emission in which an analysis is presented-in the case of Figure $1 \mathrm{H} \alpha$ emission-then one expects that distribution to be flat with a mean of 0.5 . On cumulative distribution plots, this translates to being consistent with a straight diagonal line which crosses the plot with intersections at 0,0 and 1,1 . As one moves away to the upper left of this diagonal (e.g. see the SNe Ia in Figure 1), then a population is displaying a lower degree of association to the emission. If a population falls below this diagonal line to the right, then this equates to a distribution that is biased towards the peaks of the emission, and does not simply follow the emission on a one-to-one basis. Following this we see that the $\mathrm{SNe}$ Ia show the lowest degree of association to the line emission, followed by $\mathrm{SNe} \mathrm{II}, \mathrm{SNe} \mathrm{Ib}$, and finally $\mathrm{SNe}$ Ic showing the largest degree of association. $\mathrm{H} \alpha$ emission arises from recombination of hydrogen ionised by the flux of nearby massive stars. Hence, where one observes a concentration of $\mathrm{H} \alpha$ emission, an $\mathrm{H}$ II region, one is in effect observing a concentration of young massive stars, where the dominant contributors to the ionising flux are thought to be stars with initial masses $\geq 15-20 \mathrm{M}_{\odot}$. Hence, these regions within galaxies are thought to have ages <15 Myrs (see e.g. Kennicutt 1998). The distribution of $\mathrm{H} \alpha$ flux within galaxies therefore traces ongoing SF. Hence, if different $\mathrm{SNe}$ show differences in their association to the line emission, as is shown in Figure 1, then the most straightforward interpretation of this is that we are observing differences in the mean progenitor ages, and therefore masses of SN classes. Through this interpretation, we can then order $\mathrm{SNe}$ in terms of progenitor age and mass. The results of Figure 1 imply that, as expected, $\mathrm{SNe}$ Ia have the longest progenitor lifetimes and hence the lowest masses. $\mathrm{SNe}$ II are next, and appear to have the lowest mass progenitors of the CC population, consistent with constraints from direct progenitor detections. Then follows the $\mathrm{SNe} \mathrm{Ib}$, where pixel statistics suggest a slightly higher association to ongoing SF than SNe II, however we note that this difference is marginal. The $\mathrm{SNe}$ Ic meanwhile show an almost perfect one-to-one association to $\mathrm{H} \alpha$ emission. Hence, in this interpretation we have an increasing progenitor mass sequence of: $\mathrm{SNe} \mathrm{Ia} \rightarrow \mathrm{SNe}$ II $\rightarrow \mathrm{SNe} \mathrm{Ib} \rightarrow \mathrm{SNe}$ Ic. We note that there has been considerable criticism of this simple interpretation in the literature (see e.g. Crowther 2013; Smith \& Tombleson 2015), and we return to these issues later in Section 4. However, it is important to emphasise (irrespective of interpretations) the key results from this analysis.

1. Neither $\mathrm{SNe}$ II nor $\mathrm{SNe} \mathrm{Ib}$ trace ongoing $\mathrm{SF}$ as traced by $\mathrm{H} \alpha$ emission.

2. SNe Ic accurately trace the spatial distribution of ongoing SF within hosts.

3. SNe Ib and SNe Ic show a statistically significant difference in their association with ongoing SF.

At first glance, it may seem somewhat surprising that the SN II population does not follow the spatial distribution of $\mathrm{H} \alpha$ emission, given that the latter traces high-mass SF, and that $\mathrm{SNe}$ II are the explosions of massive stars. However, as outlined above, $\mathrm{H} \alpha$ emission traces SF on timescales of $<15$ Myrs, while the progenitor population of SNe IIP has been estimated to have masses of $\sim 8-16 \mathrm{M}_{\odot}$ (Smartt et al. 2009). Hence, the latter have delay times before explosion longer 


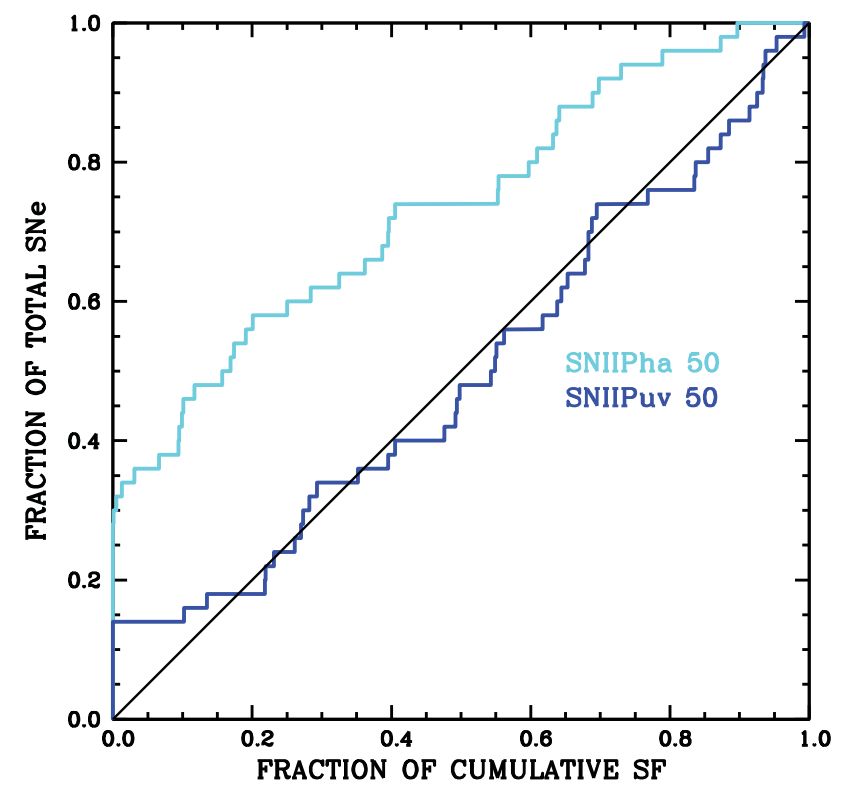

Figure 2. Cumulative $\mathrm{H} \alpha$ and near-UV NCR distributions of SNe IIP. Reproduction of Figure 4 from Anderson et al. (2012).

than the lifetimes of typical $\mathrm{H}$ II regions. Near-UV emission traces SF on longer timescales of up to $\sim 100$ Myrs (e.g. Gogarten et al. 2009b). It has been shown (Anderson et al. 2012), that indeed the SN IIP population accurately traces the near-UV emission within their host galaxies in terms of NCR pixel statistics, as is shown in Figure 2. This is completely consistent with these $\mathrm{SNe}$ arising from RSG progenitor stars at the low end of the CC SN mass range, as constrained by direct progenitor detections.

Similar work on pixel statistics has been published by other authors. Kelly et al. (2008) presented $g^{\prime}$-band pixel statistics for $\mathrm{SNe}$ and compared these to LGRBs (these authors used the fractional flux formalism of Fruchter et al. 2006, rather than the NCR method outlined above). These distributions are shown in Figure 3. While $\mathrm{H} \alpha$ line emission within galaxies is dominated by contributions from ongoing massive SF, the $g^{\prime}$-band light is a much coarser tracer of young populations. However, it is interesting that similar trends to the above are seen in terms of the relative differences between populations. SNe II follow the $g^{\prime}$-band light on an almost one-to-one basis, while the SNe Ib appear to be slightly more clustered on the brighter pixels. SNe Ic meanwhile are much more concentrated on the brightest $g^{\prime}$-band pixels (implied by the fact that the distribution falls to the bottom right of the diagonal). In absolute terms, this clustering of SNe Ic (and to a lesser extent $\mathrm{SNe} \mathrm{Ib}$ ) on the brighter $g^{\prime}$-band regions perhaps implies that massive SF is clustered with the majority of it being found at the brightest continuum ( $g^{\prime}$-band) peaks. One of the main conclusions of Kelly et al. was that SNe Ic and LGRBs have similar mass progenitor stars due to their similar associations to host galaxy young stellar populations as traced by $g^{\prime}$-band light. These same statistics were used

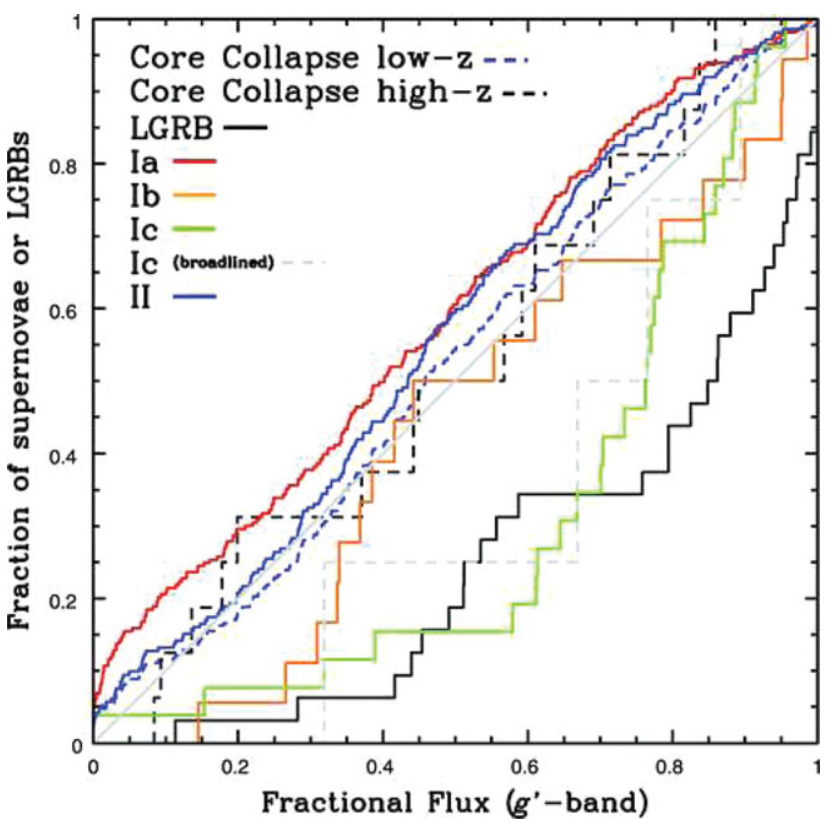

Figure 3. Cumulative $g^{\prime}$-band fractional flux distributions of $\mathrm{SNe}$ and longduration Gamma Ray Bursts. This figure is Figure 2 from Kelly et al. (2008), and is reproduced with permission of the AAS. We thank Pat Kelly for consent to use this figure.

by Raskin et al. (2008) and compared to galaxy models to constrain CC SN progenitor ages. It was concluded that the association of SNe Ic to $g^{\prime}$-band light implied a minimum progenitor mass for SNe Ic of $25 \mathrm{M}_{\odot}$.

Kangas et al. (2013) investigated specifically the local environments of $\mathrm{CC} \mathrm{SNe}$ within infrared-bright galaxies. Using NCR pixel statistics, they presented similar distributions and relative differences in distributions between SNe II and $\mathrm{SNe}$ Ic populations to the Anderson et al. results. Interestingly, these authors also presented pixel statistics with respect to other wave-bands. In particular, they showed that while SNe II did not follow the $K$-band light of their host galaxies, the $\mathrm{SNe}$ Ic do. This is surprising given that historically $K$-band light has been thought to trace the old stellar mass within galaxies. Kangas et al. suggested that this was due to the fact that in starburst environments the $K$-band light is dominated by RSGs, and hence at least in their sample is a tracer of SF. It is also interesting to note that with respect to the near-UV emission SNe Ic cluster onto the brightest pixels and show more than a one-to-one relation to the recent SF. This is also seen in Figure 19 presented below in Section 3.6.1, and may imply that the most intense SF happens in the brightest UV regions of galaxies.

Finally, we note that Crowther (2013) and Galbany et al. (2014) have also investigated the association of SNe with SF regions, through pixel statistics and by measuring distances to the nearest $\mathrm{H}$ II region. Both authors find similar statistics to those that have been presented above: $\mathrm{SNe}$ Ibc show a higher association to ongoing SF than SNe II. 


\subsection{SN radial distributions}

An updated sample of radial distributions of $\mathrm{CC} \mathrm{SNe}$ with respect to host galaxy $R$-band light was published in Habergham et al. (2012, following Anderson \& James 2009, and Habergham, Anderson, \& James 2010), also see comparisons to the radial distribution of SNe Ia in Anderson et al. 2015). That analysis concentrated on the radial distributions of the main CC SN types of SNe II, SNe Ib, and SNe Ic. It has long been observed that $\mathrm{SNe} \mathrm{Ibc}$ are more centrally concentrated within hosts than SNe II (see e.g. van den Bergh 1997 for an early example). Historically, this population difference was interpreted as a progenitor metallicity effect (which was also the initial conclusion in Anderson \& James 2009), with a higher rate of $\mathrm{SNe}$ Ibc with respect to $\mathrm{SNe}$ II in the higher metallicity central parts of galaxies. In the single star progenitor scenario, this is predicted to be the case, as more metal rich stars have stronger radiatively driven winds (e.g. Puls et al. 1996; Kudritzki \& Puls 2000; Mokiem et al. 2007), and therefore lose a greater proportion of their envelopes and hence explode as SNe Ibc rather than SNe II. This conclusion has also been further supported by global host galaxy studies, where $\mathrm{SNe} \mathrm{Ibc}$ are found to occur in more massive galaxies than SNe II (Prantzos \& Boissier 2003; Boissier \& Prantzos 2009, although see Arcavi et al. 2010 for how this is complicated when one separates $\mathrm{SNe}$ into their further subtypes), presumed to be more metal rich, and higher metallicity galaxies through direct global measurements (Prieto, Stanek, \& Beacom 2008a). However, when one separates host galaxies by their degree of disturbance, metallicity as the dominant factor in explaining radial distributions of $C C$ SNe becomes untenable.

Separating CC SN host galaxies by their degree of disturbance was first achieved by Habergham et al. (2010), and a further analysis and discussion was presented in Habergham et al. (2012). This classification separated host galaxies into three groups: undisturbed hosts, disturbed hosts, and an extreme sample which is generally dominated by major mergers (see examples of this last group in Figure 4). Galaxy classification was achieved using a series of selection criteria such as asymmetries, the appearance of a close companion, etc. The reader is referred to Habergham et al. (2012) for a full discussion. Here, we add a further $67 \mathrm{CC}$ SNe to the sample analysed in Habergham et al. (2012), including $\mathrm{SNe}$ from the Carnegie Supernova Project (CSP), where we analyse host galaxy $r^{\prime}$-band images originally obtained for light-curve host galaxy subtraction, and those $\mathrm{SNe}$ analysed in Kangas et al. (2013) who employ the same method as Anderson et al. (2012). The final sample discussed here is of $347 \mathrm{CC}$ SNe. This is split into: $226 \mathrm{SNe}$ II (where we include those SNe classified as 'II', 'IIP', and 'IIL'), $50 \mathrm{SNe}$ Ib, 65 $\mathrm{SNe}$ Ic, and a total of $121 \mathrm{SNe}$ Ibc. We exclude from this analysis both $\mathrm{SNe}$ IIb and SNe IIn (see Habergham et al. 2014 for a discussion of the radial distribution of SNe IIn).

For each SN in the above sample, we calculate an $\mathrm{Fr}_{R}$ which gives a measure of where within the radial distribution

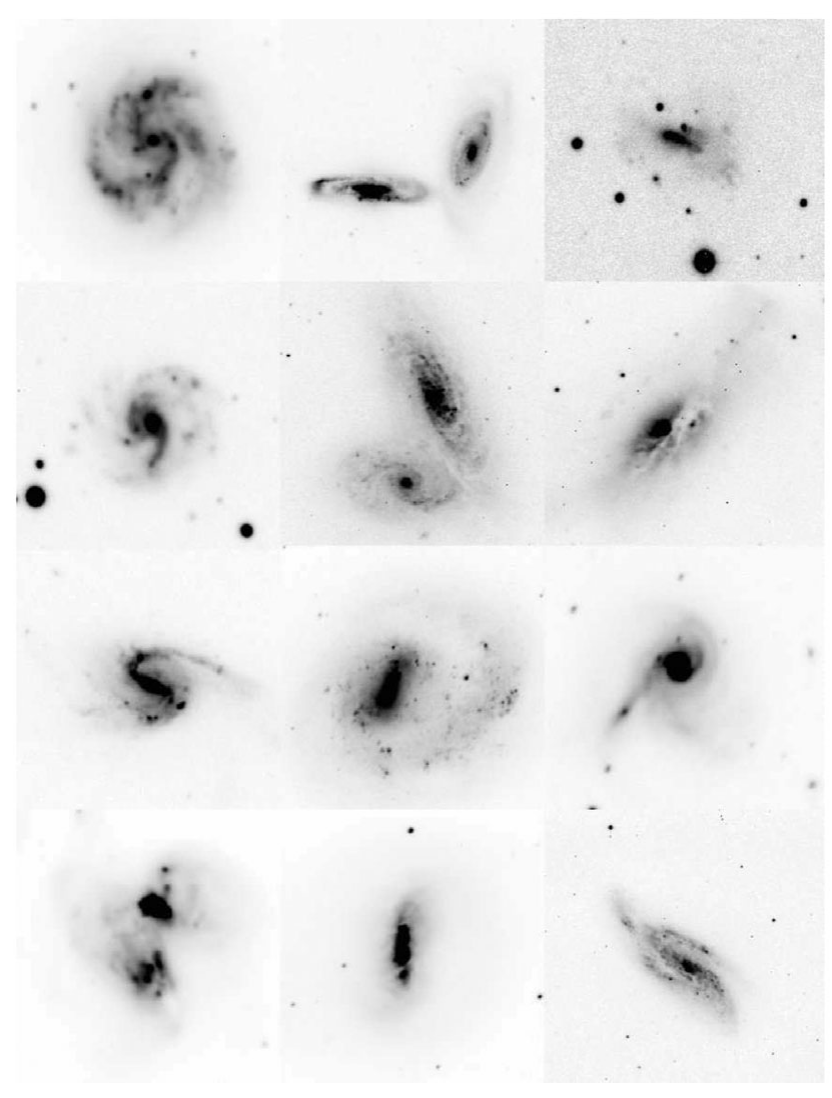

Figure 4. Examples of the most disturbed galaxy systems where CC SNe have exploded in the very central regions. This figure is Figure 5 from Habergham et al. (2010), and is reproduced with permission of the AAS.

of $R$-band light of their host galaxies they exploded. The sample is then split by the degree of disturbance, and we plot the resulting histograms and cumulative distributions in Figures 5 through 7. In Table 1, we give the mean $F r_{R}$ values for each population in each of the three galaxy samples, together with their overall values. Also presented are the KS-test statistics which give ' $D$ ' parameter indicating the amplitude of the difference between two populations and the probability ' $p$ ' that the $\mathrm{SNe}$ II and $\mathrm{SNe}$ Ibc distributions are drawn from the same parent population.

Analysing Figures 5 through 7 and Table 1, we see the following trends. In all cases, the SN Ibc population is more centralised within host continuum $R$-band light than the SNe II. However, this centralisation becomes more apparent as one moves from the undisturbed through the disturbed and finally in the extreme disturbed samples. This can be seen in the plots but also in the KS-test ' $D$ ' values in Table 1 which increase as one moves from undisturbed to disturbed host galaxy samples. The figures show that this is mainly driven by an excess of $\mathrm{SNe} \mathrm{Ibc}$ in the centres of disturbed galaxies. This is to say: as a galaxy sample becomes more disturbed, $\mathrm{SNe}$ Ibc become more centralised within their host galaxy light. It is also interesting to note the decrease in the ratio of $\mathrm{SNe}$ II to $\mathrm{SNe}$ Ibc between the galaxy samples. In the 
Table 1. CC SNe $F r_{R}$ distributions and KS-test statistics.

\begin{tabular}{lcccc}
\hline \hline SN distribution & Number of SNe & Mean $F r_{R}$ & KS-test $D$ & KS-test $p$ \\
\hline Undisturbed sample & & & & \\
$\quad$ SNe II & 118 & 0.578 & & \\
$\quad$ SNe Ibc & 51 & 0.483 & 0.224 & 0.048 \\
Disturbed sample & & & & \\
$\quad$ SNe II & 85 & 0.489 & & 0.023 \\
$\quad$ SNe Ibc & 51 & 0.376 & 0.259 & \\
Extreme sample & 23 & 0.507 & & \\
$\quad$ SNe II & 19 & 0.347 & 0.380 & 0.074 \\
$\quad$ SNe Ibc & & & & \\
\hline \hline
\end{tabular}

In column 1, the SN distribution type is listed, followed by the number of events analysed in column 2 . Then, the mean $\mathrm{Fr}_{R}$ value is listed for each distribution. For each set of two distributions, the KS-test ' $D$ ' and probability value ' $p$ ' are listed in columns 4 and 5 respectively.

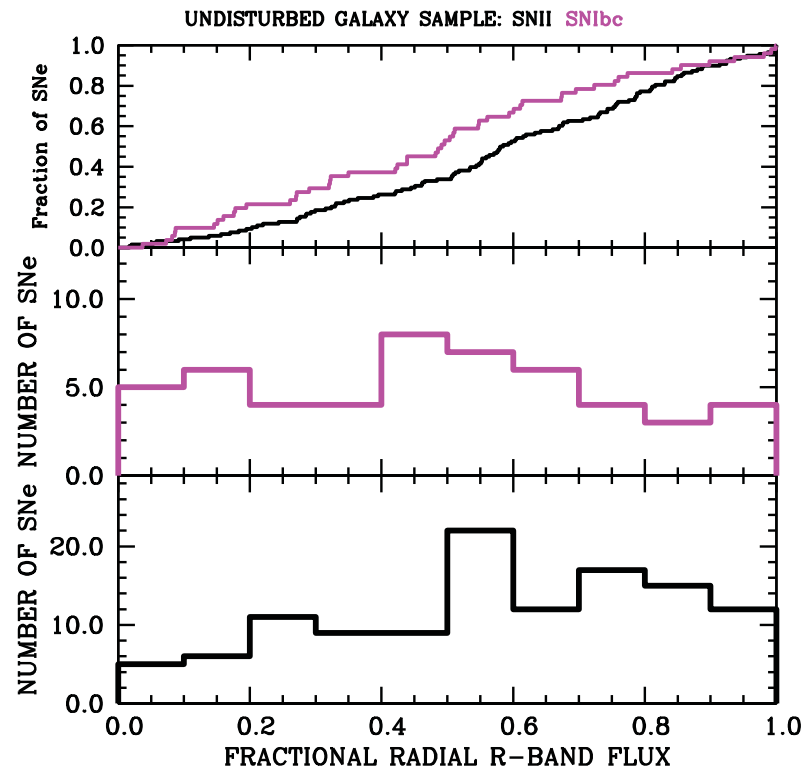

Figure 5. Histograms of the $\mathrm{Fr}_{R}$ distributions of CC SNe in undisturbed galaxy systems. In the bottom panel, the SN II population is presented, and in the middle panel the SNe Ibc. In the top panel, the cumulative distributions of both populations are presented.

undisturbed sample the ratio is 2.3 , then 1.7 in the undisturbed sample, and finally 1.2 in the extreme sample, i.e. the number of $\mathrm{SNe} \mathrm{Ibc}$ to $\mathrm{SNe}$ II increases significantly as the degree of host galaxy sample disturbance increases. This is consistent with previous work (Habergham et al. 2010, 2012; Hakobyan et al. 2014).

Throughout this review we show that there exist significant differences in the environments of $\mathrm{SNe} \mathrm{Ib}$ and $\mathrm{SNe} \mathrm{Ic}$. In Figure 8, we present the $F r_{R}$ distributions for the individual $\mathrm{SN} \mathrm{Ib}$ and SN Ic populations when the galaxy sample is split into undisturbed and disturbed galaxies (an extreme sample is not discussed here due to the low number of events in that distribution). In Table 2, the mean values and KS-test statistics between these samples are listed. It is observed that here the difference between populations is more pronounced in the undisturbed samples (while above the difference between $\mathrm{SNe}$ II and the overall SN Ibc samples was more pronounced

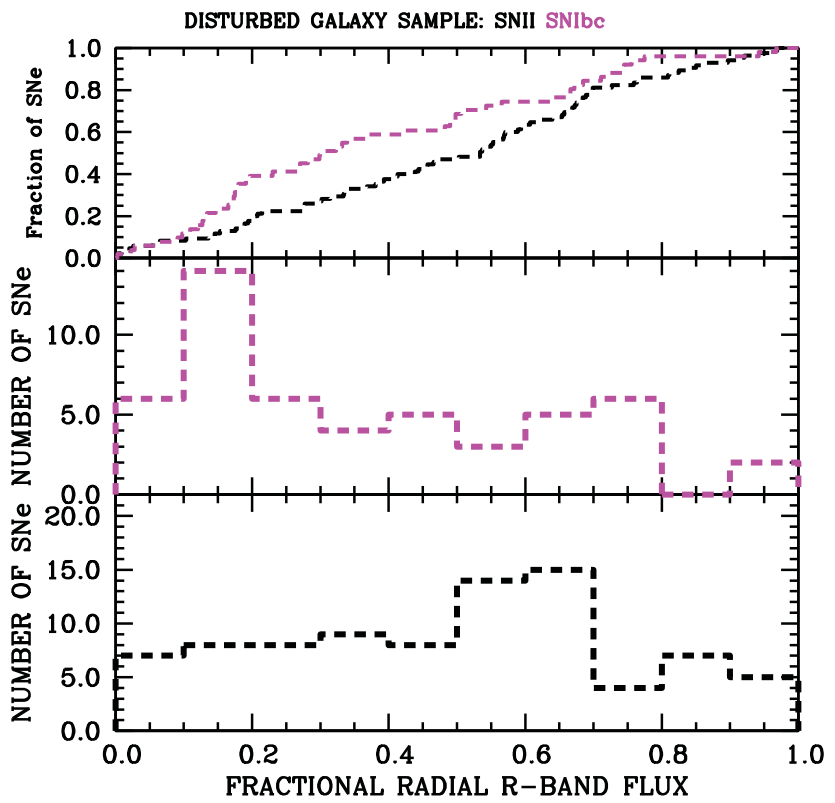

Figure 6. Histograms of the $F r_{R}$ distributions of $\mathrm{CC} \mathrm{SNe} \mathrm{in} \mathrm{disturbed}$ galaxy systems. In the bottom panel, the SN II population is presented, and in the middle panel the SNe Ibc. In the top panel, the cumulative distributions of both populations are presented.

in the disturbed and extreme samples). SNe Ic are found to occur more centrally than SN Ibc in both undisturbed and disturbed galaxy samples, however the effect is significantly larger in the former.

While SNe Ibc are more centrally concentrated than SNe II in disturbed systems, the explanation of this finding is not simple, and it is not clear that metallicity effects are responsible. While metallicity gradients are observed in most galaxies, they can become much weaker or even non-existent in disturbed/merging galaxies (see e.g. Kewley et al. 2010 and most recently Sánchez et al. 2014). During the process of interaction, pristine gas flows to the inner parts of the system, enriching the central parts of galaxies with low abundance material (see e.g. Hibbard \& van Gorkom 1996; Rampazzo et al. 2005; Kewley, Geller, \& Barton 2006). In other words, metallicity gradients are stronger in undisturbed 
Table 2. SN Ib and SN Ic $F r_{R}$ distributions and KS-test statistics.

\begin{tabular}{lcccc}
\hline \hline SN distribution & Number of SNe & Mean $F r_{R}$ & KS-test $D$ & KS-test $p$ \\
\hline Undisturbed sample & & & & \\
$\quad$ SNe Ib & 20 & 0.594 & 0.393 & 0.042 \\
$\quad$ SNe Ic & 27 & 0.390 & & \\
Disturbed sample & 30 & 0.437 & 0.262 & 0.322 \\
$\quad$ SNe Ib & 38 & 0.348 & \\
SNe Ic & & & & \\
\hline \hline
\end{tabular}

In column 1, the SN distribution type is listed, followed by the number of events analysed in column 2 . Then the mean $F r_{R}$ value is listed for each distribution. For each set of two distributions, the KS-test ' $D$ ' and probability value ' $p$ ' are listed in columns 4 and 5 respectively.

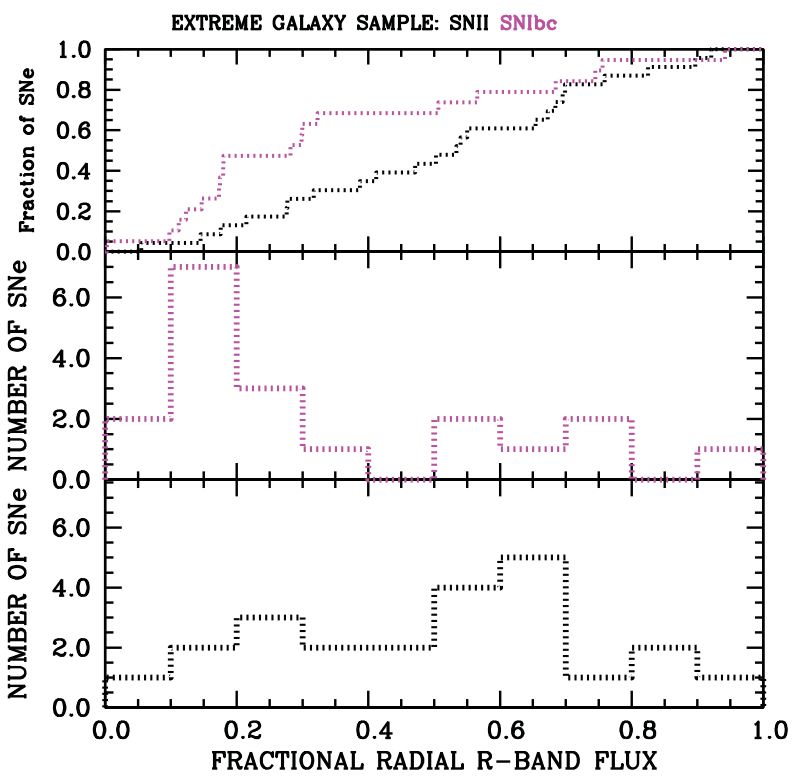

Figure 7. Histograms of the $F r_{R}$ distributions of CC SNe in the most disturbed galaxy systems. In the bottom panel, the SN II population is presented, and in the middle panel the SNe Ibc. In the top panel, the cumulative distributions of both populations are presented.

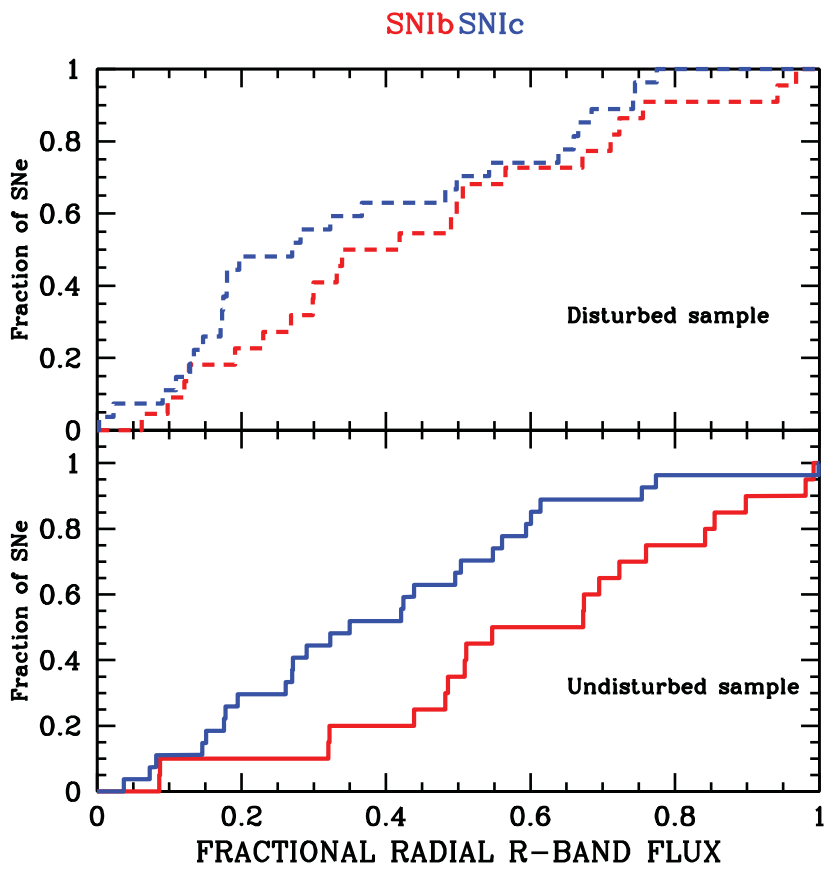

Figure 8. Cumulative $F r_{R}$ distributions for $\mathrm{SNe} \mathrm{Ib}$ and $\mathrm{SNe}$ Ic after host galaxies have been split into undisturbed and disturbed samples. (Note, here we do not plot the separate extreme sample due to a lack of statistics, but this also forms part of the disturbed sample).

1. Differences between the distributions of $F r_{R}$ values of $\mathrm{SNe}$ II and $\mathrm{SNe}$ Ibc increase as one moves from undisturbed to disturbed host galaxy samples.

2. In disturbed/interacting systems, $\mathrm{SNe} \mathrm{Ibc}$ are much more centrally concentrated within galaxies than $\mathrm{SNe}$ II.

3. There is a central excess of SNe Ibc in the central regions of disturbed galaxies compared to host galaxy continuum light.

4. Differences in the radial distributions of CC SNe cannot be dominated by progenitor metallicity effects.

5. However, in the undisturbed sample SNe Ic are seen to be more centrally concentrated than $\mathrm{SNe} \mathrm{Ib}$, hinting at a metallicity effect between these two sub-types. 
In Habergham et al. (2010) and Habergham et al. (2012), it was speculated on how these results can thus be understood after discounting progenitor metallicity effects. Indeed, it was suggested that the centralisation of SNe Ibc in disturbed systems could indicate that in these dense violent SF processes induced in the central regions of these galaxies, that the initial mass function (IMF) is biased to the production of more massive stars, and hence a higher proportion of $\mathrm{SNe} \mathrm{Ibc}$ to $\mathrm{SNe}$ II is produced. It is also possible that in the central regions of these galaxies there is a higher fraction of interacting binaries, and hence a higher number of SN Ibc explosions. Both of these possibilities were also considered in the radial analysis of Hakobyan et al. (2009), and are further discussed below.

Kangas et al. (2013) also analysed CC SN host galaxies using the same radial analysis as discussed above. These authors concentrated on $\mathrm{SNe}$ within infrared bright galaxies (many of which are starbursts), finding very similar results our analysis where we combine their sample into our large radial analysis database. A further interesting result from Kangas et al. is that the SNe II in their sample were found to prefer to explode in the outer regions of the $K$-band light of their hosts.

\subsection{Host $\mathrm{H}$ II region metallicities}

The first statistical study of SN environmental metallicities concentrated on probing differences between BL SNe Ic that had or had not accompanied LGRBs, finding lower oxygen abundances for the latter (Modjaz et al. 2008). This suggests that low metallicity is an important parameter in producing these extreme events. In Anderson et al. (2010), a comparison was made between $\mathrm{SNe}$ II and $\mathrm{SNe} \mathrm{Ibc}$ environment oxygen abundances. It was concluded that any implied progenitor metallicity difference between these SN types was relatively small. That study also suggested that the difference between $\mathrm{SNe} \mathrm{Ib}$ and $\mathrm{SNe}$ Ic $\mathrm{H}$ II region metallicities was small. However, this was contested by Modjaz et al. (2011), who claimed a difference in that SNe Ic arise from regions of higher oxygen abundance. These findings were interpreted as evidence for more complete envelope-stripping in high-metallicity progenitors, indicating a dominant role for line-driven winds. Leloudas et al. (2011) also presented an analysis of $\mathrm{SNe}$ Ibc environment oxygen abundances finding something in between those two previous studies. A sample of SNe Ibc formed from exclusively non-targeted SN searches was presented by Sanders et al. (2012). Those authors concluded that only a small difference between $\mathrm{SNe} \mathrm{Ib}$ and $\mathrm{SNe}$ Ic environment oxygen abundance existed, and also analysed previous work in the literature to conclude that any difference between $\mathrm{SNe}$ II and $\mathrm{SNe} \mathrm{Ibc} \mathrm{H}$ II region oxygen abundances was small. A sample of SNe II from the untargeted search program PTF (Palomar Transient Factory, Rau et al. 2009) was published by Stoll et al. (2013). This study looked in detail at the properties of their sample, and found that it had a very similar oxygen abundance distribution to
SN II samples drawn from galaxy targeted surveys (i.e. that in Anderson et al. 2010). This is somewhat surprising, as it is generally assumed that a sample drawn from targeted searches will be biased towards $\mathrm{SNe}$ in more massive, metalrich host galaxies, compared with those found within untargeted rolling searches. Most recently Kuncarayakti et al. (2013a) and Kuncarayakti et al. (2013b) published SN II and $\mathrm{SN}$ Ibc samples of $\mathrm{H}$ II region oxygen abundances, where they focussed on very nearby events and analysed integral field unit (IFU) spectroscopy, attempting to identify specific parent stellar clusters. Again, they found only marginal evidence for an implied metallicity difference between the two $\mathrm{SN}$ samples, although $\mathrm{SNe}$ Ic were found to occur in regions of higher oxygen abundance than $\mathrm{SNe} \mathrm{Ib}$.

In light of the significant number or studies above, we proceed to present a meta-analysis of all CC SN H II region oxygen abundance measurements. We also include unpublished values from the CALIFA survey, which will be presented in L. Galbany et al. (in preparation). In our final sample, we combine the unpublished values from CALIFA with those from: Modjaz et al. (2008), Thöne et al. (2009), Anderson et al. (2010), Modjaz et al. (2011), Leloudas et al. (2011), Anderson, Habergham, \& James (2011), Prieto et al. (2012), Habergham et al. (2012), Van Dyk et al. (2012), Sanders et al. (2012), Stoll et al. (2013), Tomasella et al. (2013), Kuncarayakti et al. (2013a, 2013b), Inserra et al. (2013), and Taddia et al. (2013b) $)^{3}$. Our final sample is of 245 CC SN host H II region oxygen abundances. Here, we only choose to analyse SNe II (120 SNe, where this includes SNe IIP, SNe IIL, and those events classified as simply SNe II in the literature), $\mathrm{SNe} \mathrm{Ib}$ (56) and SN Ic (59, with a total of $125 \mathrm{SNe} \mathrm{Ibc})$, to be consistent with the previous pixel statistics and radial analyses presented above. It is noted that here we include data from many different sources, where data reduction and spectral extraction may be different between samples. These samples also come from different SN search sources, including targeted and un-targeted searches, which again brings issues such as differences in relative ratios of SN types. However, our aim here simply to bring all the relevant data together in one place, and use significant statistics to investigate whether there indeed exist any statistical differences in implied host $\mathrm{H}$ II region metallicities.

In Figure 9, we present the cumulative oxygen abundance distributions of the main CC SN types. All mean values, and KS-test $D$ and $p$ values are listed in Table 3. It is observed that while essentially all distributions have statistically very similar implied environment metallicities, the SN Ic population has the highest mean value. Statistically speaking, we find no evidence for environment and hence progenitor metallicity differences between SNe II and the overall SN Ibc population. We note that there is a suggestion that $\mathrm{SNe}$ Ic have higher metallicities than $\mathrm{SNe} \mathrm{Ib}$, but this is not statistically significant. In Figure 10, we show the ratio of SNe II to SNe Ibc as

\footnotetext{
${ }^{3}$ We include all measurements that can be considered of the 'environment' of individual SNe. In practice this means that we use values extracted at 'the nearest $\mathrm{H}$ II region', in addition to those at exact explosion sites.
} 


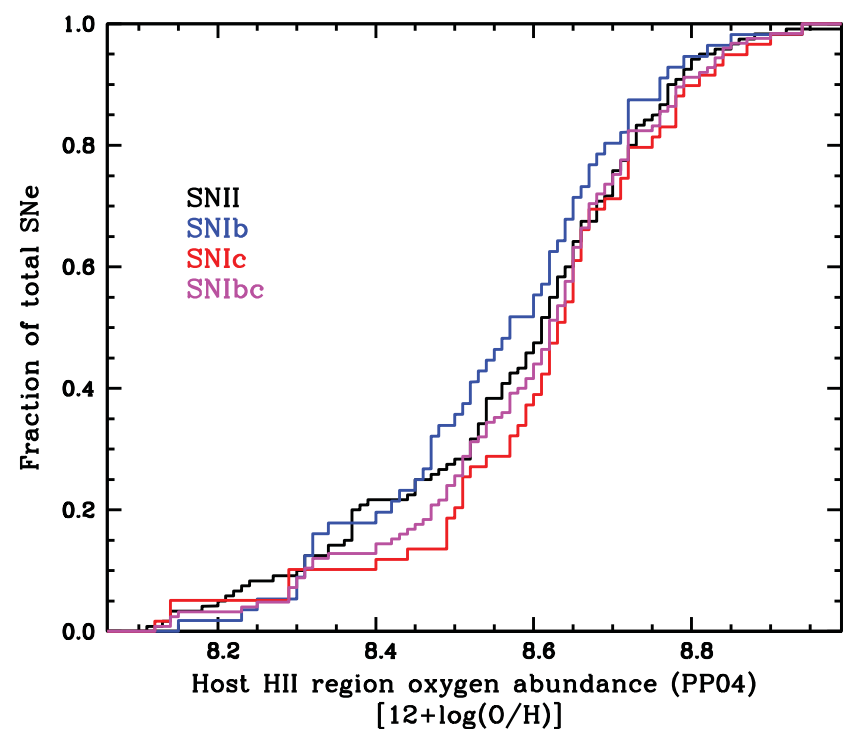

Figure 9. Cumulative distributions of $\mathrm{CC} \mathrm{SN}$ host $\mathrm{H}$ II region oxygen abundance. Here, we show the SN II, SN Ib, SN Ic, and the combined SN Ibc populations.

Table 3. CC SN H II region oxygen abundances and KS-test statistics.

\begin{tabular}{lcc}
\hline \hline SN distribution type & Number of SNe & Mean $12+\log (\mathrm{O} / \mathrm{H})$ \\
\hline SNe II & 120 & 8.57 \\
SNe Ibc & 125 & 8.59 \\
SNe Ib & 56 & 8.56 \\
SNe Ic & 59 & 8.61 \\
\hline SN populations & $D$ & $p$ \\
\hline SNe II-Ibc & 0.09 & $\gg 10 \%$ \\
SNe Ib-Ic & 0.21 & $\sim 15 \%$ \\
SNe Ib-II & 0.11 & $\gg 10 \%$ \\
SNe Ic-II & 0.13 & $\gg 10 \%$ \\
\hline \hline
\end{tabular}

In the top panel in column 1, the SN distribution type is listed, followed by the number of events analysed in column 2 . Then the mean $Z$ value is listed for each distribution. In the bottom panel, column 1 indicates the two distributions being compared, and columns 2 and 3 list the KS-test $D$ and $p$ values between those distributions.

a function of host $\mathrm{H}$ II region oxygen abundance. Here, we simply split the sample into five equally-sized bins (in terms of numbers of $\mathrm{SNe}$ ). Errors on ratios are Poissonian. The ratio is essentially flat with metallicity, with some suggestion that the SN Ibc/SN II ratio decreases in the lower bin (which has a mean $12+\log (\mathrm{O} / \mathrm{H})$ of 8.29). In Figure 11, we further split the sample and show how the ratios: SN Ib/II, SN Ib/Ic, and $\mathrm{SN}$ Ic/II change as a function of host $\mathrm{H}$ II region oxygen abundance. The $\mathrm{SN} \mathrm{Ib/Ic} \mathrm{ratio} \mathrm{appears} \mathrm{to} \mathrm{increase} \mathrm{slightly}$ with decreasing metallicity. The SN Ic/II ratio is flat with oxygen abundance, while the $\mathrm{SN} \mathrm{Ib} / \mathrm{II}$ ratio appears to increase slightly. Again, we emphasise the low significance of all of these trends. We list the important results from this CC SN H II region metallicity analysis:

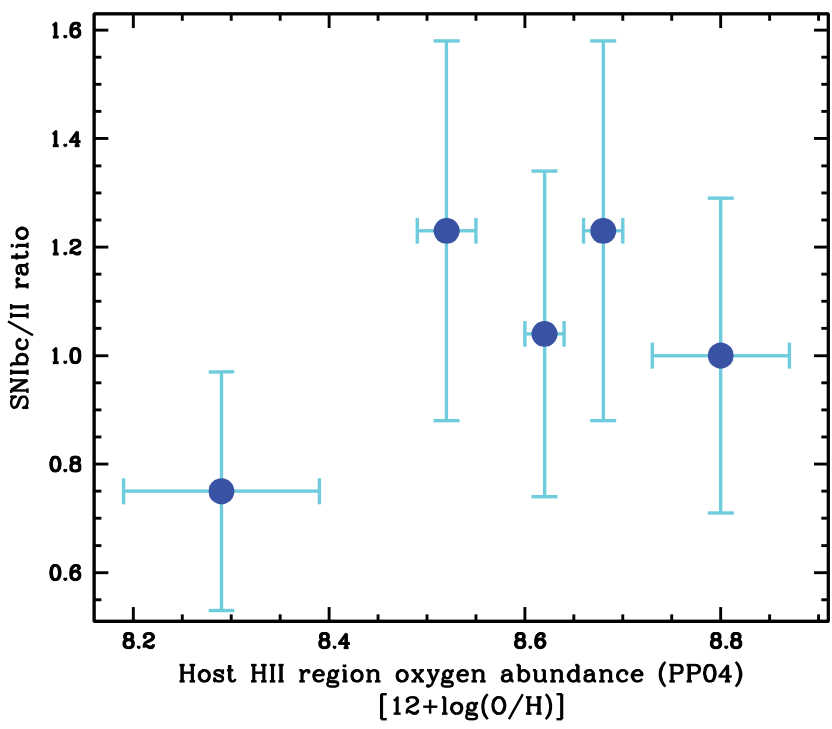

Figure 10. The ratio of SN Ibc to SN II events as a function of host $\mathrm{H}$ II region oxygen abundance. The full sample of $\mathrm{CC} \mathrm{SNe}$ are simply split into five bins of equal size (in terms of number of measurements) of oxygen abundance.

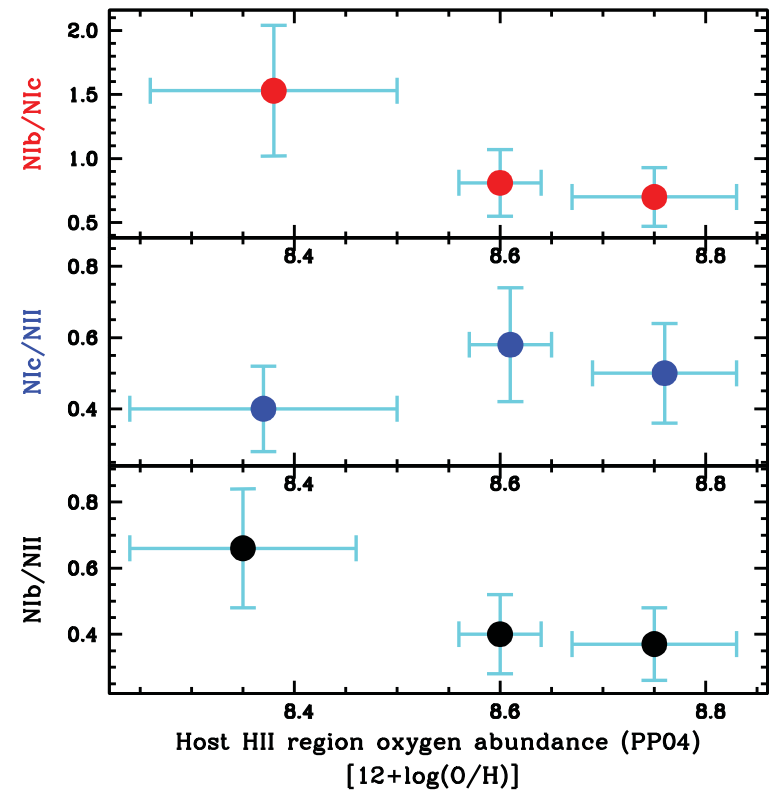

Figure 11. Top: the ratio of $\mathrm{SNe} \mathrm{Ib}$ to $\mathrm{SNe}$ Ic as a function of host $\mathrm{H}$ II region oxygen abundance; Middle: the ratio of SNe Ic to SNe II; Bottom: the ratio of SNe Ib to SNe II. In all three histograms, the samples are simply split into three equal-sized bins of oxygen abundance.

1. Overall we find only small, statistically insignificant differences in the environment oxygen abundances of $\mathrm{SNe}$ II, SNe Ib, and SNe Ic.

2. There is a suggestion that the ratio of $\mathrm{SNe}$ II to $\mathrm{SNe} \mathrm{Ibc}$ increases in the lowest metallicity bin.

3. The SN Ib to SN Ic ratio appears to increase with decreasing oxygen abundance. 
These results suggest that progenitor metallicity is not a driving parameter in producing the majority of CC SN diversity. This obviously does not rule out metallicity playing a part in producing specific SN sub-types, or changing the properties of more specific transient features (other than simple type classifications). However, this does seem to suggest that other parameters such as progenitor mass and/or the presence and influence of a binary companion are much more dominant. Indeed, given that single stellar models (see Heger et al. 2003; Eldridge \& Tout 2004; Georgy et al. 2009; Ibeling \& Heger 2013) predict a strong dependence SN type production with respect to progenitor metallicity, the above results (and those published in the literature previously, see Anderson et al. 2010; Sanders et al. 2012 for combined SNe II and $\mathrm{SNe}$ Ibc analyses) indicate that a significant fraction of $\mathrm{CC}$ $\mathrm{SNe}$ are the result of massive star binary interactions. We return to this conclusion in Section 4.

\subsection{Further environment studies}

In this section, we summarise and discuss CC SN environment studies which do not fall easily into previous sections. IFU spectrographs allow one to obtain both spatial and spectral information simultaneously. In Section 5, we discuss the future of environment studies where we argue that IFUs will play a dominant role, following surveys such as CALIFA (Sánchez et al. 2012), and using new facilities such as the recently commissioned MUSE instrument at the VLT (Bacon et al. 2010). Kuncarayakti et al. (2013b) and Kuncarayakti et al. (2013a) presented IFU environment studies of $11 \mathrm{SNe}$ II and $13 \mathrm{SNe}$ Ibc respectively. They used the power of IFU observations to first identify the nearest stellar cluster to $\mathrm{SN}$ explosion positions, before extracting the spectrum at that position. Environment oxygen abundances were then estimated using the same strong emission line diagnostics as outlined above finding similar distributions for $\mathrm{SNe}$ II and SNe Ibc. These authors also measured $\mathrm{H} \alpha \mathrm{EWs}$ which they used to trace the age of the local stellar population, which can then be translated to progenitor masses using stellar evolution models. In Figure 12, we reproduce the mass-metallicity figure from Kuncarayakti et al. (2013b), where measurements were compared to both model predictions and SN II direct progenitor detections. It was concluded that a number of SNe Ibc were associated with stellar clusters with ages consistent with relatively low progenitor masses, where the implied progenitor scenario would be binary systems. In addition, in some cases relatively high progenitor masses were derived for SNe II. In Figure 13, we reproduce the $\mathrm{H} \alpha \mathrm{EW}$ distributions from Kuncarayakti et al. (2013b), which show differences between SNe IIP, SNe IIL, SNe Ib, and SNe Ic. While the statistics are limited, it appears that $\mathrm{H} \alpha$ EWs are generally similar between SNe IIP and SNe Ibc, with some suggestion that those for SNe Ic are slightly larger, possibly consistent with the $\mathrm{H} \alpha$ pixel statistics presented above. The EWs of SNe IIL appear to have a tendency for very large values, implying high-mass progenitors. However, we

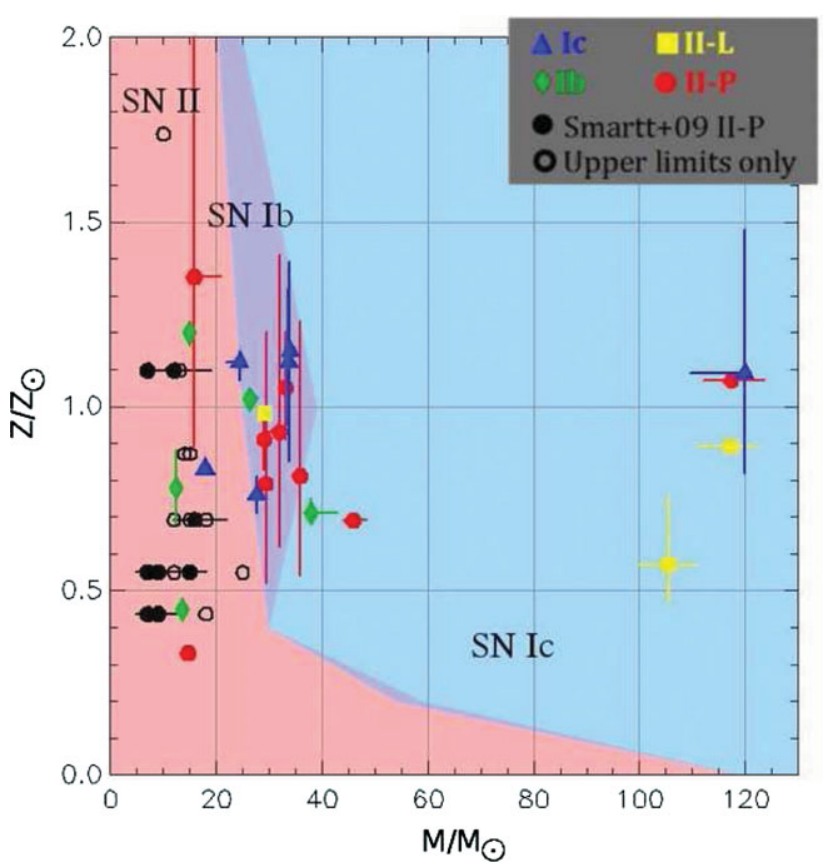

Figure 12. Progenitor age against progenitor metallicity plot. SN environment ages and metallicities are derived using IFU spectroscopy. These are compared to a model grid taken from Georgy et al. (2009), and also SN II direct progenitor detections from Smartt et al. (2009). This figure is Figure 17 taken from Kuncarayakti et al. (2013b) and is reproduced by permission of the AAS.

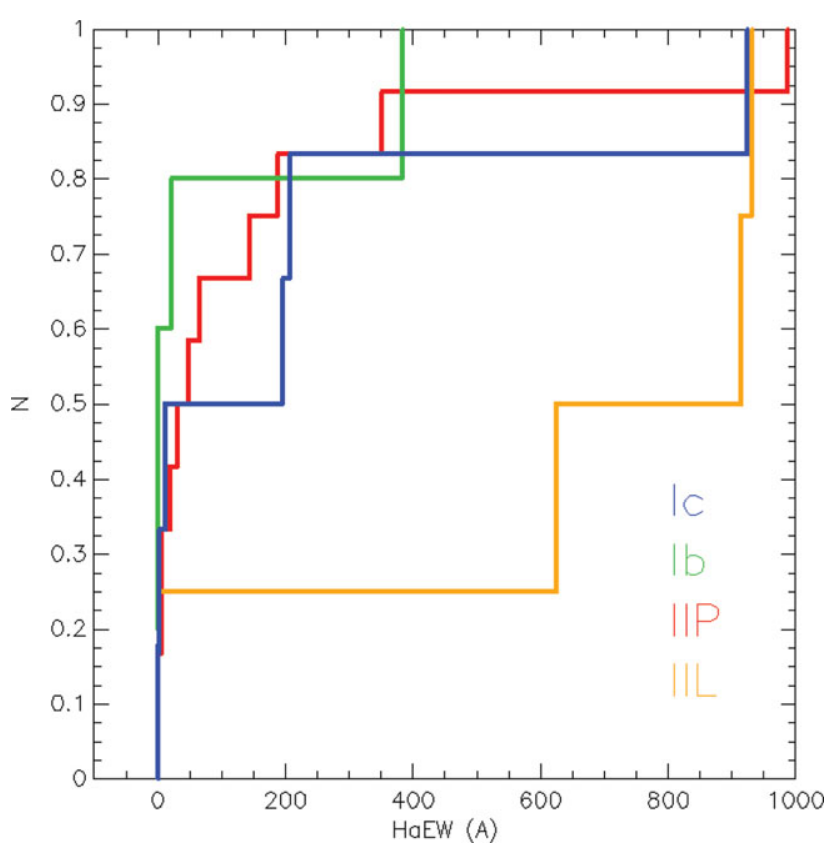

Figure 13. $\mathrm{H} \alpha$ EWs of the stellar population observed within the parent stellar clusters of CC SNe, derived from IFU spectroscopy. SN IIP, IIL, Ib, and Ic distributions are presented. A larger EW indicates a younger age. This figure is an edited version of Figure 18 taken from Kuncarayakti et al. (2013b) and is reproduced by permission of the AAS. 


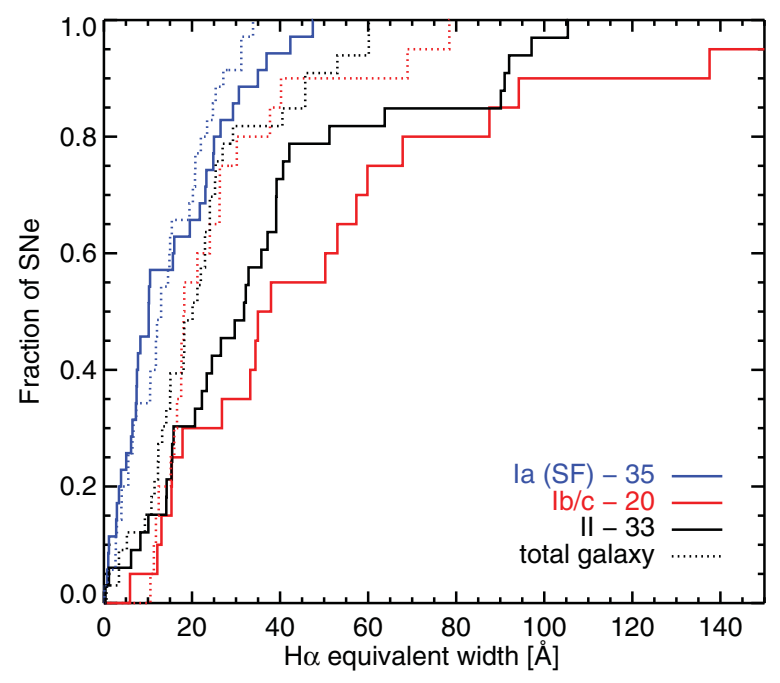

Figure 14. $\mathrm{H} \alpha \mathrm{EWs}$ of the stellar population observed at the explosion sites of SNe, derived from IFU spectroscopy. SN Ia, II, and Ibc distributions are presented. This figure is part of Figure 13 taken from Galbany et al. (2014) and is reproduced by permission of A\&A.

caution that these studies are currently limited by low statistics, where issues such as associating a particular cluster to a parent progenitor population may become problematic. A significant number of new observations have been obtained for this study, and results from a larger sample promise to be enlightening. The Kuncarayakti et al. studies concentrate on very nearby SNe where one is resolving individual stellar clusters. Galbany et al. (2014) used lower resolution IFU observations to also measure $\mathrm{H} \alpha \mathrm{EWs}$ at the explosion sites of $\mathrm{SNe}$ (and in addition analysed various measures of the association of SNe to SF region, see below). In Figure 14, $\mathrm{H} \alpha$ EW distributions for SNe Ia, SNe II, and $\mathrm{SNe}$ Ibc are displayed. As expected, SN Ia environments have the lowest EW distribution. It is interesting that here the $\mathrm{SNe} \mathrm{Ibc}$ appear to explode in regions of slightly higher EW than SNe II, indicating younger ages, and therefore slightly higher masses for the former. Again, larger samples and further analysis are needed to build on these studies.

Another distinct environment analysis which has yet to be discussed, is multi-colour photometric analysis. Kelly \& Kirshner (2012) presented a large statistical analysis of 519 CC SN environments deriving various parameters for the unresolved stellar populations found at explosion sites. In Figure 15, we present their environment $u^{\prime}$-band surface brightness against $u^{\prime}-z^{\prime}$ colour plot. SNe Ic BL (broad-line) and $\mathrm{SNe} \mathrm{IIb}$ were observed to explode in significantly bluer regions of their hosts than other $\mathrm{SN}$ types, while the $u^{\prime}$-band surface brightness of SN Ic and SN Ib environments was observed to be higher than those of SNe II, again somewhat consistent with the $\mathrm{H} \alpha$ pixel statistics above. These differences in environments suggest some differences in progenitor properties such as age or mass (possibly related to $u^{\prime}$-band surface brightness) and metallicity (relating to environment colour). Another interesting result from Kelly \& Kirshner
(2012) was that no significant difference was found in the environment properties of SNe IIn and the 'normal' SN II population. This argues for similar progenitor properties, a conclusion that will be further strengthened below. Kelly \& Kirshner presented a number of other interesting environment and global host galaxy measurements, and the reader is encouraged further to explore that publication.

\subsection{SN Ia environments}

This review concentrates on the environmental properties of $\mathrm{CC} \mathrm{SNe}$, as those have been the most widely studied. However, especially in the most recent years work has been published on the local environments of SNe Ia. Given the much longer expected delay times of SNe Ia, interpreting environmental results becomes more difficult as progenitors have possibly travelled large distances before explosion. However, some intriguing results have been published.

Raskin et al. (2009) used a modified version of the Fruchter et al. (2006) fractional flux method to analyse SN Ia environment pixel statistics, comparing observations to analytical galaxy models, concluding that even the 'prompt' component of the SN Ia population has delay times of several 100 Myrs. Stanishev et al. (2012) presented a first IFU study of SN host galaxies, analysing the spatial and spectral information obtained for a sample of six SNe Ia, and showing the capabilities of using such observations. Rigault et al. (2013) also used IFU observations of a large sample of SN Ia host galaxies. These authors concentrated on measuring the strength or presence of $\mathrm{H} \alpha$ emission at the explosion sites of SNe Ia. They found that $\mathrm{SNe}$ where emission was indeed detected have redder colours than those where no emission is detected (a similar result was also found by Anderson et al. 2015, see below). Wang et al. (2013) speculated that two distinct populations of SN Ia progenitors exist, with those events having higher ejecta velocities being more centrally-concentrated than their lower-velocity counterparts. However, this result has recently been questioned in an independent study by Pan et al. (2015). Rigault et al. (2013) showed that SNe Ia associated with local $\mathrm{H} \alpha$ emission are more homogeneous and give a lower dispersion when used as distance indicators (also see Rigault et al. 2015). Subsequent work by Kelly et al. (2015) identified a sub-set of SNe Ia which exploded in environments of high UV surface brightness and SF surface density, which gave significantly more accurate distance measurements than $\mathrm{SNe}$ Ia in all environments.

In Anderson et al. (2015), the measurements described in Sections 2.1 and 2.2 are used to study a sample of $\sim 100$ literature SNe Ia. Host galaxy NCR pixel statistics were obtained from near-UV through optical and near-IR imaging. It was found that the SN Ia population in star-forming galaxies most accurately follows the spatial distribution of host $B$-band light, as shown in Figure 16. This implies that the dominant progenitor population of SNe Ia in star-forming galaxies has neither very long delay times, i.e. they do not follow the near-IR $J$ - or $K$-band light, nor very short delay 


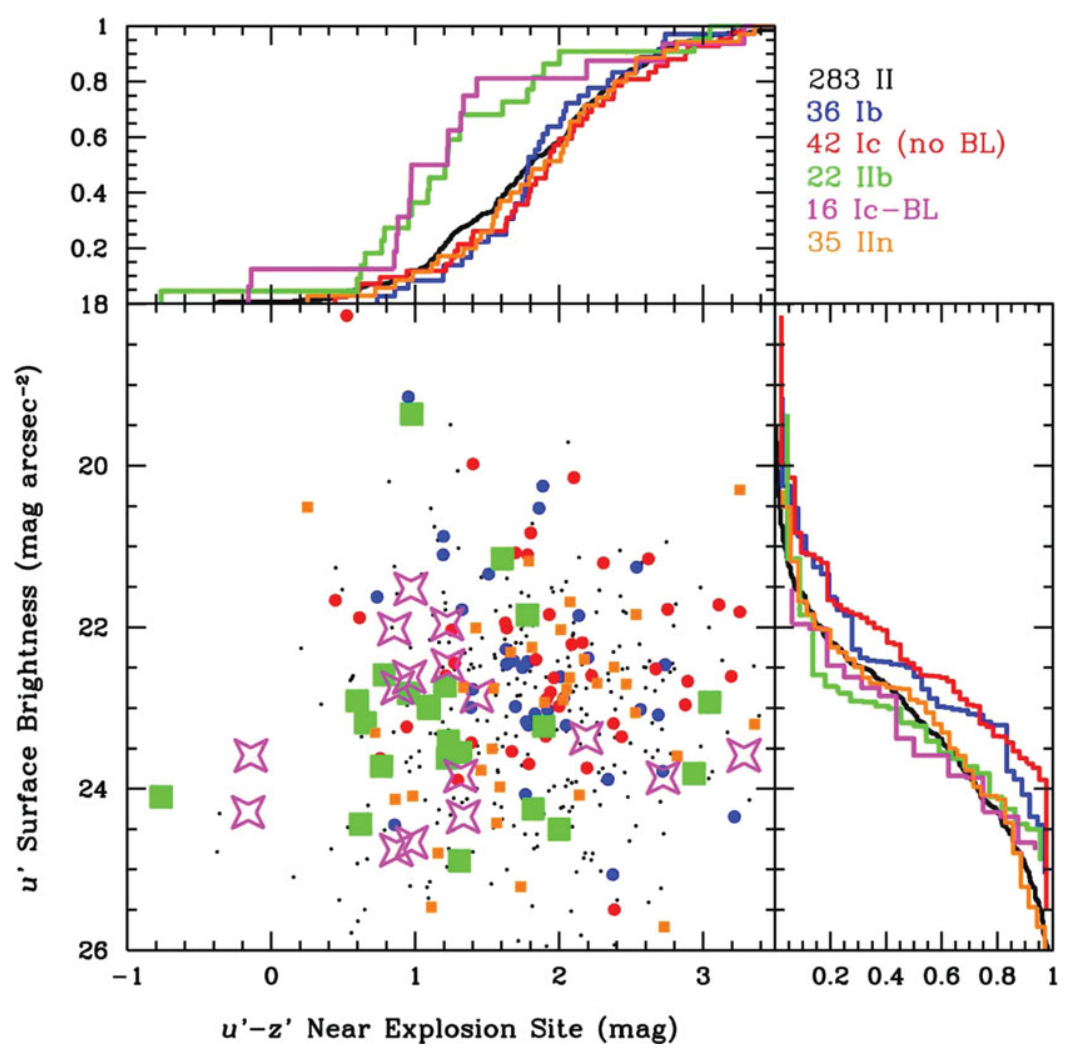

Figure 15. $u^{\prime}$-band surface brightness against $u^{\prime}-z^{\prime}$ colours of CC SN environments. The larger inset shows the two parameters plotted against each other, while above and to the right are the cumulative distributions of each property. This figure is Figure 2 taken from Kelly \& Kirshner (2012), and is reproduced by permission of the AAS. We thank Pat Kelly for consent to use this figure.

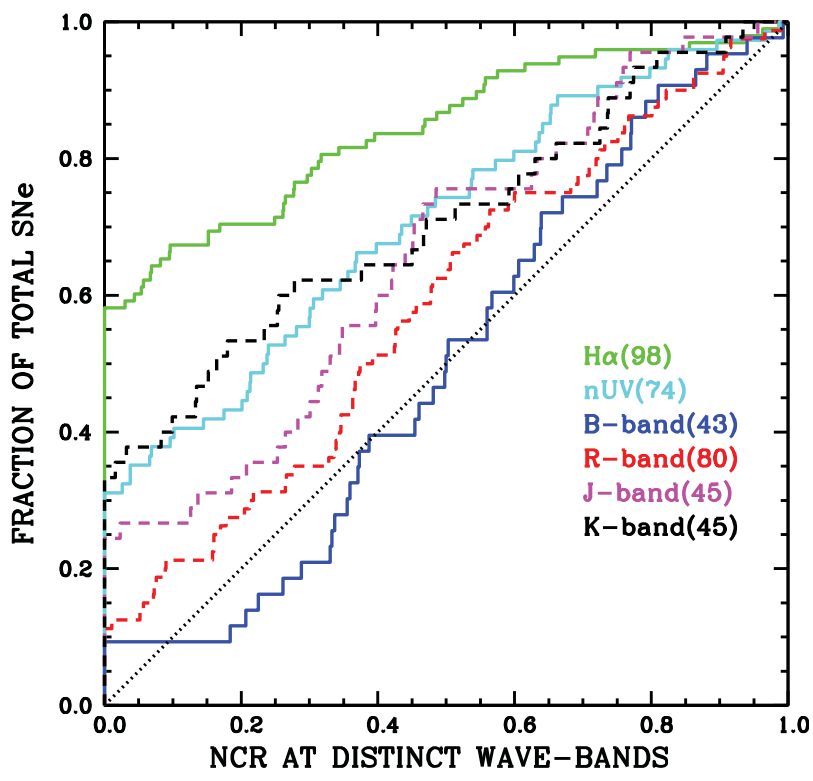

Figure 16. Cumulative NCR pixel distributions for SNe Ia at distinct waveband observations: $\mathrm{H} \alpha$, near-UV, $B-, R-, J$ - and $K$-band. This figure is taken from Anderson et al. (2015). times, i.e. they do not follow the near-UV emission. In addition, it is shown in Figure 17 that when the analysed SN Ia sample is split by SN colour at maximum, 'redder' events are more likely to be associated with $\mathrm{H} \alpha$ emission that their 'blue' counterparts. This hints at host galaxy extinction driving the colour diversity of SNe Ia. The reader is encouraged to read that paper in full for an overall view of SN Ia environments.

\subsection{The environments of SN sub-types}

Above we have focussed discussion on the environments of the main SN types. In such studies, one needs sufficient statistics to enable comparisons between different SN populations to be enlightening. However, some studies have looked at (observed) rarer $\mathrm{SN}$ types and studied their environments with the aim of providing progenitor constraints.

\subsubsection{Interacting transients}

SNe IIn have historically been assumed to arise from extremely massive stars, given the large amounts of CSM material implied from their interaction-driven spectra. Indeed, it has been speculated (e.g. Smith 2008) that their progenitors are luminous blue variable stars (LBVs). In addition, in 


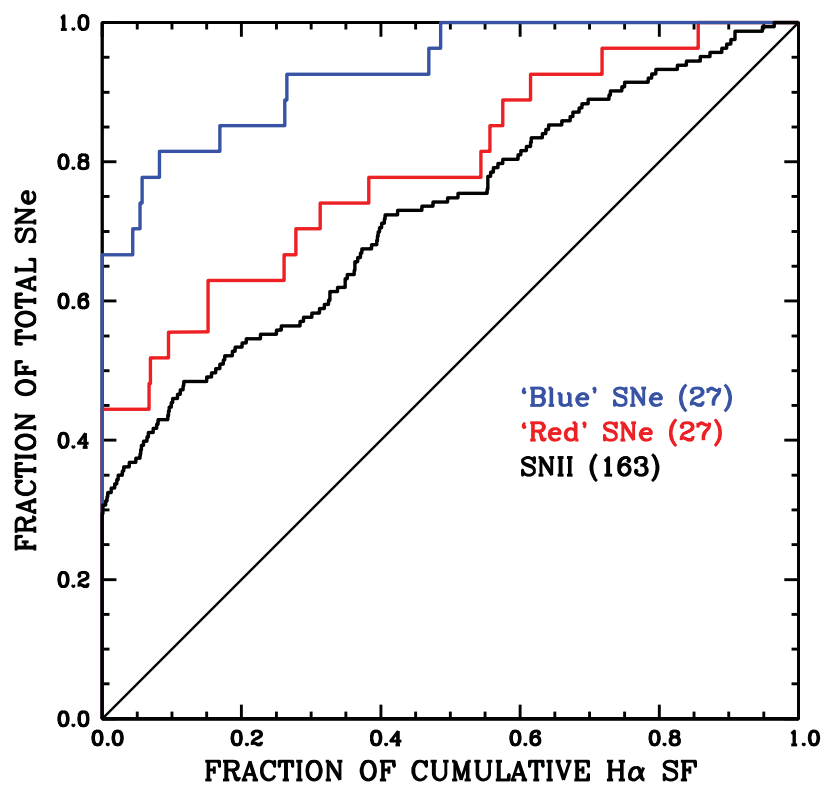

Figure 17. Cumulative $\mathrm{H} \alpha \mathrm{NCR}$ distributions for $\mathrm{SNe}$ Ia when the sample is split by $\mathrm{SN}$ colour at maximum light. The SN II distribution is also shown for reference. This figure is taken from Anderson et al. (2015).

the case of SN $2005 \mathrm{gl}$ the progenitor star was constrained to be a very massive and probable LBV star from detection on pre-explosion images (Gal-Yam \& Leonard 2009). In the case of the transient SN 2009ip (more details on the environment of this intriguing event below), pre-eruption data appear to suggest a very luminous, very massive star. If very massive star progenitors were the case for all SNe IIn, then one would expect their explosion sites to be similar to the environments of very massive stars or other $\mathrm{SNe}$ expected to arise from such massive star populations. In Figure 18, we plot the cumulative $\mathrm{H} \alpha \mathrm{NCR}$ distributions of SNe IIn compared to those of other SN types, as taken from Habergham et al. (2014). The surprising result is observed that SNe IIn show the lowest degree of association with host galaxy $\mathrm{H} \alpha$ emission of all CC SN types. This would appear to suggest that $\mathrm{SNe}$ IIn have lower progenitor masses than previously assumed. A specific analysis of SN IIn environments is presented in Habergham et al. (2014). In Figure 19, cumulative NCR pixel statistics are shown with respect to host galaxy near-UV emission. This shows that the SNe IIn accurately trace the recent SF within their host galaxies, in a very similar fashion to SNe IIP. The similarity of the distributions of SNe IIn and SNe IIP with respect to both $\mathrm{H} \alpha$ and nearUV emission, suggests that their progenitor lifetimes, and hence masses are similar. In addition, in Figures 18 and 19 we also plot the SN Ic population. The SN Ic distributions are clearly offset from that of SNe IIn, and if we assume that $\mathrm{SNe}$ Ic arise from the highest progenitor masses of $\mathrm{CC}$ $\mathrm{SNe}$, this is further evidence that SN IIn have relatively lower mass progenitors (we also note the differences in their radial distributions seen in Habergham et al. 2014). Another interesting aspect of Figure 19 is that SNe Ic show a higher

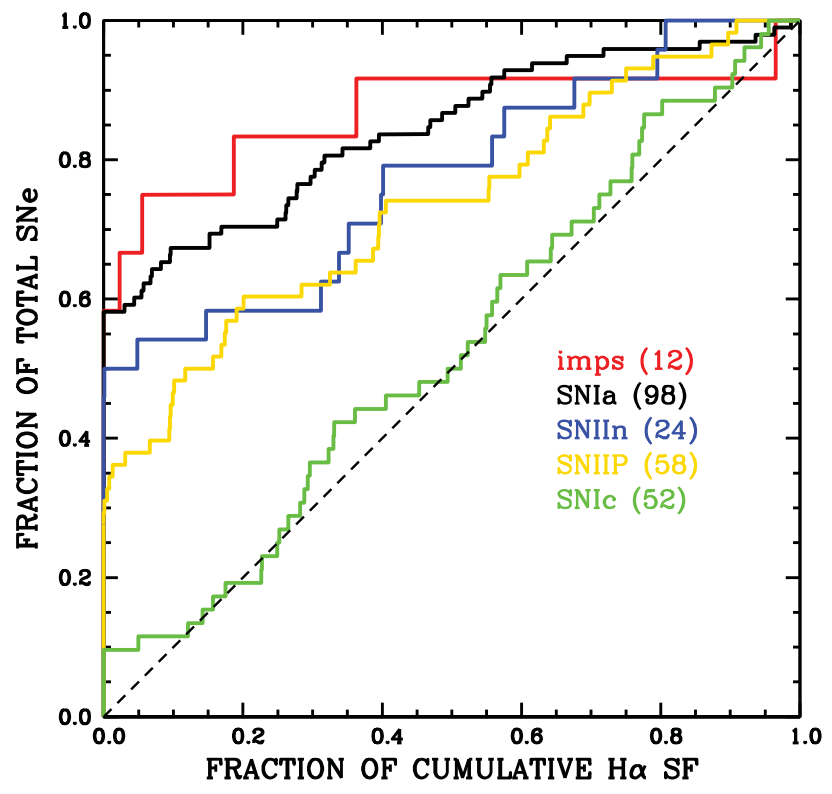

Figure 18. Cumulative $\mathrm{H} \alpha$ NCR distributions of the interacting transients SNe IIn and 'impostors' as compared to SNe Ia, SNe II, and SNe Ic. This figure is Figure 5 taken from Habergham et al. (2014).

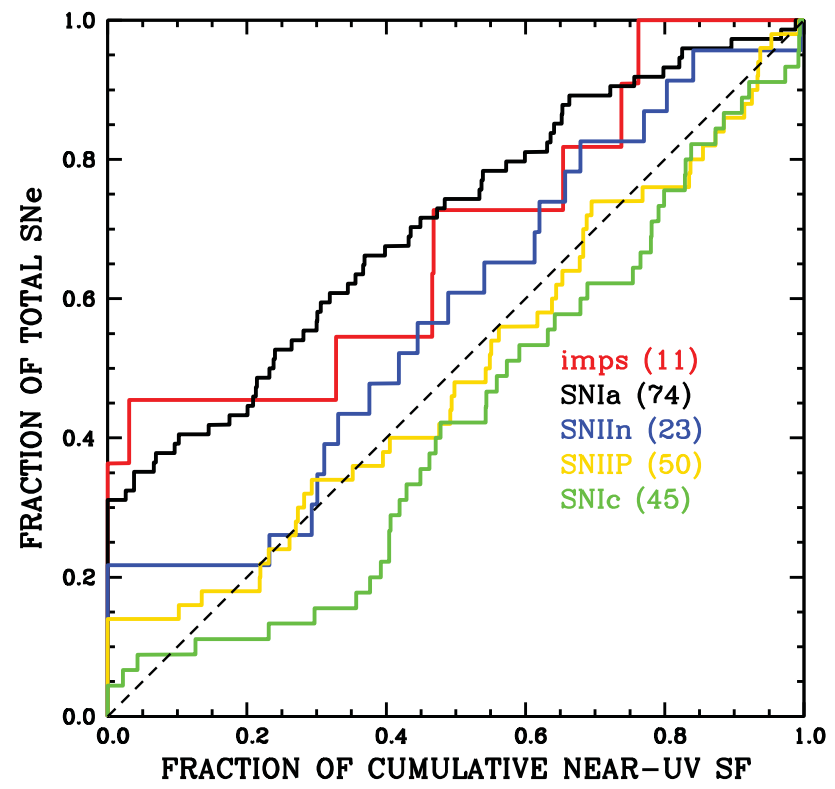

Figure 19. Cumulative near-UV NCR distributions of the interacting transients SNe IIn and 'impostors' as compared to SNe Ia, SNe II, and SNe Ic. This figure is Figure 6 taken from Habergham et al. (2014).

than one-to-one association to the near-UV emission. This is consistent with what was previously observed by Kelly et al. (2008) investigating SN pixel statistics with respect to host galaxy $g^{\prime}$-band light and again implies that the most massive $\mathrm{SF}$ is clustered to the brightest UV regions within galaxies.

There are a number of transients discovered where it is not clear whether they are the deaths of stars, or a large-scale eruption which produces a bright (but significantly dimmer than most $\mathrm{SNe}$ ) transient. These transients have spectra very 
similar to SNe IIn, and are named SN 'impostors' (see van Dyk et al. 2000; Maund et al. 2006, and Kochanek, Szczygieł, \& Stanek 2012 for a recent discussion). The environments of these events were also analysed and discussed in Habergham et al. (2014), and their pixel statistics are shown in Figures 18 and 19. Here, we find that 'impostors' show an even lower degree of association to SF within galaxies than SNe IIn. However, it is important to note that the much lower luminosities of 'impostors' compared to other SNe means that significant selection effects could be at play in the discovery of these transients against bright $\mathrm{H}$ II regions.

SN 2009ip is a transient with an unclear nature. Indeed, it is still debated whether the star has finally terminally exploded or that the transient is still being powered by eruptions and interactions (Smith et al. 2010; Prieto et al. 2013; Pastorello et al. 2013; Mauerhan et al. 2013; Fraser et al. 2013; Margutti et al. 2014; Smith, Mauerhan, \& Prieto 2014; Graham et al. 2014; Mauerhan et al. 2014). One extremely interesting aspect of this transient that is often overlooked is the region within its host galaxy where it exploded, i.e. its environment. As outlined in particular in Fraser et al. (2013), SN 2009ip occurred in the very outer regions of its host galaxy, more than $4 \mathrm{kpc}$ from the galaxy centre, with the vast majority of the host galaxy light being contained within the isophotal ellipse that passes through the $\mathrm{SN}$ position (indeed, Fraser et al. 2013 estimated an $F r_{R}$ value of $\sim 1$ ). This is shown in Figure 20, reproduced from Fraser et al. (2013). While it has been shown in Habergham et al. (2014) that SNe IIn and 'impostors' are generally found to more frequently explode within the outer regions of hosts than e.g. SNe IIP, SN 2009ip is still at the extreme of these distributions. The explosion site of SN 2009ip is somewhat surprising given that there are strong indications that the progenitor was or is a very massive star of $>60 \mathrm{M}_{\odot}$ (Foley et al. 2011). However, there is no sign of ongoing or recent SF in the immediate vicinity of SN 2009ip, and neither is there any discernible spiral arm near to the explosion location (Fraser et al. 2013). If the progenitor of SN 2009ip was such a massive star, as seems to be the case, then its environment is puzzling. It may be that this transient is simply an example of extreme isolated SF. It is also possible that the radial position of the explosion implies a low-metallicity environment and hence progenitor. For now, the environment of SN 2009ip remains a mystery; however, it will be intriguing whether such isolated regions within galaxies are typical of these types of events, once a statistical analysis is possible in the future.

\subsubsection{SNe IIb}

As noted in the introduction, SNe IIb are transitional events that first show signs of hydrogen (hence the 'II'), which then fades and the $\mathrm{SNe}$ appear similar to $\mathrm{SNe} \mathrm{Ib}$. Their relative rarity dictates that statistical studies of their environments lack significant numbers. However, some interesting trends have been observed. In Anderson et al. (2012), it was found that SNe IIb appeared to accurately trace host galaxy SF, and there was a suggestion that the degree of association

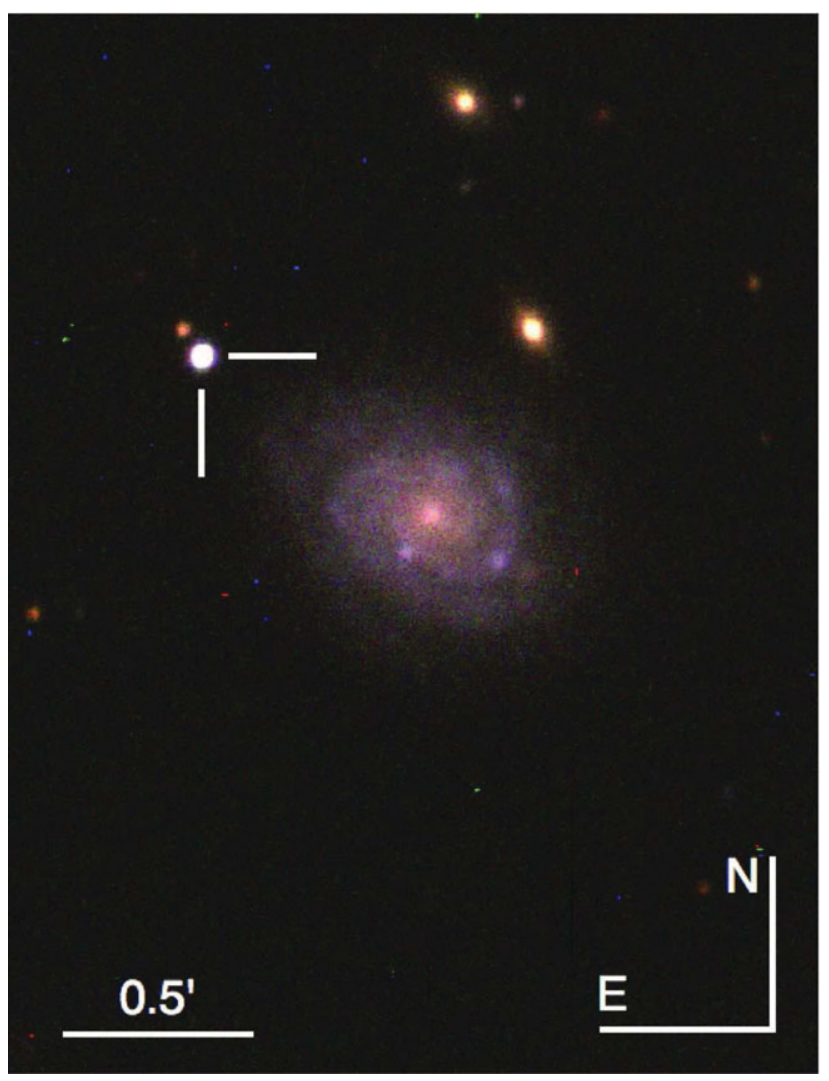

Figure 20. The environment location of SN 2009ip within its host galaxy. This figure is a reproduction of Figure 22 from Fraser et al. (2013).

was higher than that of SNe Ib. In Figure 15, it can be seen that $\mathrm{SNe}$ IIb have significantly bluer environments than most other CC SNe. In addition, there is some suggestive evidence that $\mathrm{SNe}$ IIb prefer to explode in lower metallicity environments than the majority of other CC SNe (Modjaz et al. 2011; Sanders et al. 2012). Hence, the environments of SNe IIb do show differences to those of other $\mathrm{CC} \mathrm{SNe,} \mathrm{which} \mathrm{may} \mathrm{give}$ clues as to their specific progenitor properties. However, additional statistics are needed before concrete conclusions can be made on progenitors.

\subsubsection{SNe Ic BL}

A sub-set of SNe Ic are known as $\mathrm{SNe}$ Ic BL, as their spectra show significantly broader features, indicating significantly higher ejecta velocities (and hence more energetic explosions). These events are of particular interest because of their link to LGRBs (Woosley \& Bloom 2006). Studies of their environments have shown that SNe Ic BL usually explode in low-metallicity environments, in particular less metal rich than 'normal' SNe Ic (Modjaz et al. 2011; Sanders et al. 2012). However, those SNe Ic BL which accompany LGRBs appear to explode in even lower metallicity environments than SNe Ic BL without LGRBs (see Modjaz et al. 2008 and Modjaz et al. 2011). As shown in Figure 15, Kelly \& Kirshner (2012) demonstrated that SNe Ic BL, like the SNe IIb above, explode in blue environments, indicated by the 
$u^{\prime}-z^{\prime}$ colour at the explosion site. Kelly \& Kirshner (2012) speculated that for both SNe IIb and SNe Ic BL that the blue environments are linked to the low metallicity of those same locations.

\subsubsection{A-like events}

Taddia et al. (2013a) studied specifically the environments of SNe with similar properties to SN 1987A. SN 1987A is the closest $\mathrm{SN}$ observed in the modern era of astronomy. SN 1987A was peculiar in that its light curve showed a slow extended rise (see e.g. Arnett et al. 1989). There are now a handful of similar SNe which have been discovered and studied (Pastorello et al. 2005; Kleiser et al. 2011; Pastorello et al. 2012; Taddia et al. 2012; Arcavi et al. 2012). SN 1987A itself exploded in the Large Magellanic Cloud (LMC) at a distance of only $50 \mathrm{kpc}$. The wealth of information from pre-explosion observations enabled detailed studies of its progenitor star which was observed to be a blue supergiant star. Given the unusual nature of this $\mathrm{SN}$, it is interesting to ask whether these peculiarities are related to the specific environment within which it exploded.

Taddia et al. (2013a) studied the environments of 13 87Alike SNe using very similar methods to those outlined above. Figure 21, from Taddia et al. (2013a), shows the cumulative distributions of the $87 \mathrm{~A}$-like population with respect to other CC SNe in terms of: host galaxy absolute magnitudes; galactocentric radial positions; and host $\mathrm{H}$ II region metallicities. It appears that 87A-like SNe prefer environments with somewhat lower metallicities than other $\mathrm{CC} \mathrm{SNe}$, as indicated directly by the measured oxygen abundances of their host $\mathrm{H}$ II regions, and confirmed by their fainter host galaxy luminosities and higher radial distances within these hosts. Taddia et al. also investigated NCR values for their 87A-like sample, finding a similar distribution to SNe IIP.

\subsubsection{SNe Iax}

SNe Iax is the term proposed by Foley et al. (2013) for a set of SNe which have peculiar features that strongly distinguish them from all other classes of SNe. While they are spectroscopically similar to SNe Ia, they have significantly fainter peak magnitudes and lower expansion velocities at maximum light. Indeed, it is still not completely clear whether these events are thermonuclear or $\mathrm{CC}$ in nature. Investigations into their environments seem to imply relatively young progenitors. Lyman et al. (2013) showed that SNe Iax have a similar degree of association to host galaxy ongoing SF as SNe IIP, and used this to argue for progenitors with delay times of less than 50 Myrs. It is interesting to note that Foley et al. (2014) constrained the progenitor lifetime of the extreme SN Iax SN 2008ha to be less than 80 Myrs. Meanwhile, McCully et al. (2014) presented pre-explosion data of the SNe Iax SN 2012Z, and speculated that the progenitor was a WD accreting matter from a helium star, however they could not rule out a massive star origin. While the interpretation of these detections remains unclear, both appear to point towards relatively short-lived progenitors for SNe Iax, consistent with their environmental properties.

\subsubsection{Ca-rich events}

SN 2005E was the first SN to be termed 'Calcium-rich' (Carich), by Perets et al. (2010). A larger sample of these events have now been presented (see Perets et al. 2011; Kasliwal et al. 2012; Valenti et al. 2014). Ca-rich events are usually spectroscopically classified as $\mathrm{SNe}$ Ibc, but are significantly fainter and present unusually strong calcium features. As above for the SNe Iax, it has been debated whether these events arise from massive star explosions (see e.g. Kawabata et al. 2010), or thermonuclear events. The strange locations of these events was noted for SN 2005E (Perets et al. 2010) and SN 2005cz (Perets et al. 2011), with both events occurring in non-star-forming galaxies, and in the case of SN 2005E very far from their host galaxy. Indeed, in the compilation of Kasliwal et al. (2012), these remote locations with large offsets from host galaxies were shown to be common. Yuan et al. (2013) compared the locations of Ca-rich events with results from cosmological simulations, concluding that their progenitors are likely to be of low metallicity and very old age. Lyman et al. (2013) showed that the association of Carich events with host galaxy SF is similar to that of SNe Ia, again favouring significantly delayed progenitors. Most recently, Lyman et al. (2014b) presented deep Hubble Space Telescope imaging of the explosion sites of Ca-rich events. The lack of any evidence of an underlying stellar population at the sites of these explosions led these authors to conclude that $\mathrm{Ca}$-rich $\mathrm{SNe}$ are in fact high-velocity kicked systems, which have moved with significant velocities from their birth sites, hence explaining their environment properties.

So far, we have not discussed differences in the environments of SNe IIP and SNe IIL. Results have indeed been published on the environments of SNe IIL (see e.g. Anderson et al. 2012; Kuncarayakti et al. 2013b). However, type IIP/IIL classifications in the literature are extremely subjective. In the next section, we present discussion on relating environment properties to exact light-curve and spectral measurements of SNe II.

Finally, we note that we have not discussed the environments of super-luminous SNe (SLSNe). While such studies are certainly interesting, most of the published work thus far has concentrated on global host properties (see e.g. Neill et al. 2011; Lunnan et al. 2014a; Leloudas et al. 2015; Chen et al. 2014), although a few very recent studies have investigated their immediate environments (Lunnan et al. 2014b; Thöne et al. 2014). It will be interesting in the future to apply the same methods as used above to study these mysterious explosions.

\subsection{Correlating specific transient features with environment properties}

To date, environment and host galaxy studies have generally concentrated on investigating differences in the 

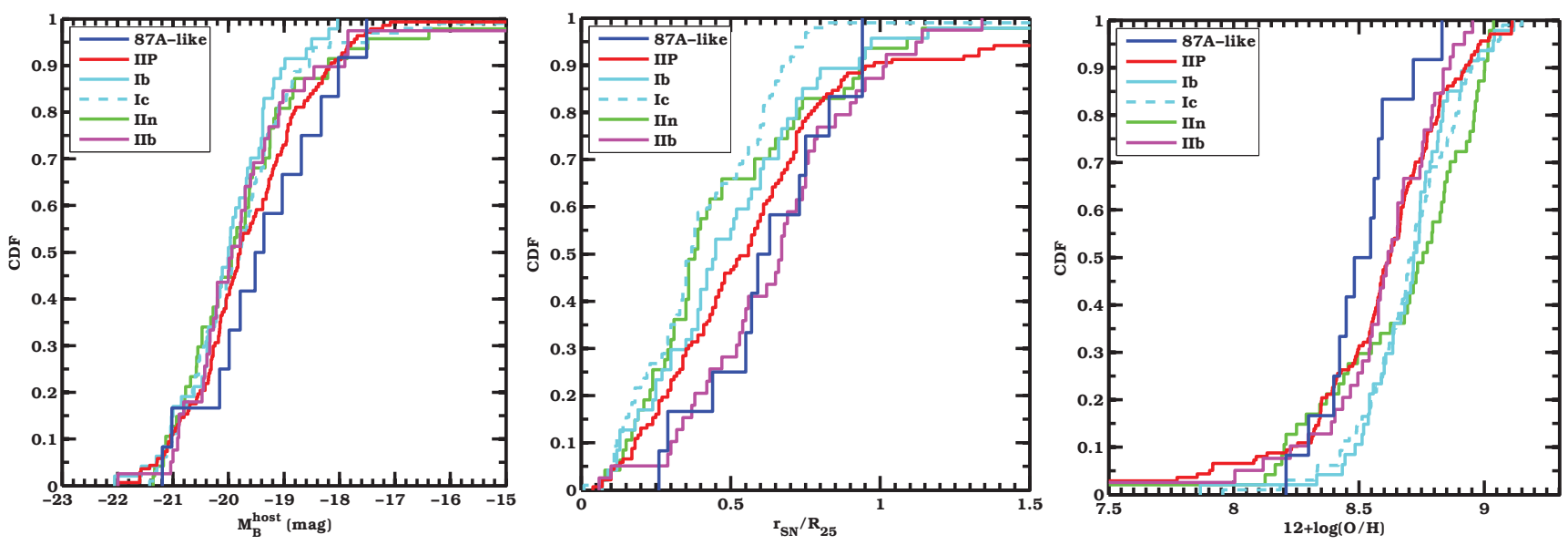

Figure 21. Comparison of the environments of 87A-like events with other CC SNe. Left: cumulative host galaxy absolute magnitude distributions for CC SNe. Middle: cumulative $R_{25}$ normalised galactocentric radial distributions of CC SNe. Right: cumulative host $\mathrm{H}$ II region oxygen abundance distributions for CC SNe. This figure is of Figure 1 from Taddia et al. (2013a) and is reproduced by permission of A\&A.

environmental properties of SNe when events are simply split by spectroscopic type. These typings are often somewhat subjective, and in the case of SNe IIP and SNe IIL, spectroscopic typing without any further light-curve information used can simply be wrong. This is an issue with SN catalogues which are often not updated after initial classifications are given in circulars. Indeed, it has been shown in Gutiérrez et al. (2014) that while correlations between SN II spectral parameters and light-curve morphologies do exist, there is significant dispersion, which can lead to ambiguous spectroscopic classifications. In the case of $\mathrm{SNe} \mathrm{IIb}$ and $\mathrm{SNe} \mathrm{Ib}$, the epoch at which the classification spectrum is taken can then lead to an event being typed into one class or the other (see e.g. Milisavljevic et al. 2013 for discussion on this issue). Given these ambiguities, it makes sense for future statistical studies to use more specific SN features to investigate environment trends. While historically this type of analysis was difficult due to the lack of large $\mathrm{SN}$ follow-up databases, recent years have seen a number of publications of statistically significant samples of CC SNe (SN Ia large samples have been relatively more widespread, as is seen in the analysis in Section 3.5). SN II photometry, spectra and measured parameters for large samples have been recently published in Arcavi et al. (2012), Anderson et al. (2014a, 2014b), Gutiérrez et al. (2014), Faran et al. (2014b), Sanders et al. (2015), and Faran et al. (2014a). SNe Ibc and SNe IIb samples have been published in Drout et al. (2011), Bianco et al. (2014), and Modjaz et al. (2014). This provides an opportunity to correlate environment properties to more specific features such as light-curve morphologies, the presence and form of specific spectral features and their evolution in time, and parameters that have a direct link to explosion and progenitor properties such as synthesised nickel mass.

In this section, we analyse the environmental properties of the SN II sample presented and analysed in Anderson et al. (2014b). That work presented a sample of more than 100 well sampled $V$-band light-curves from which key pa-

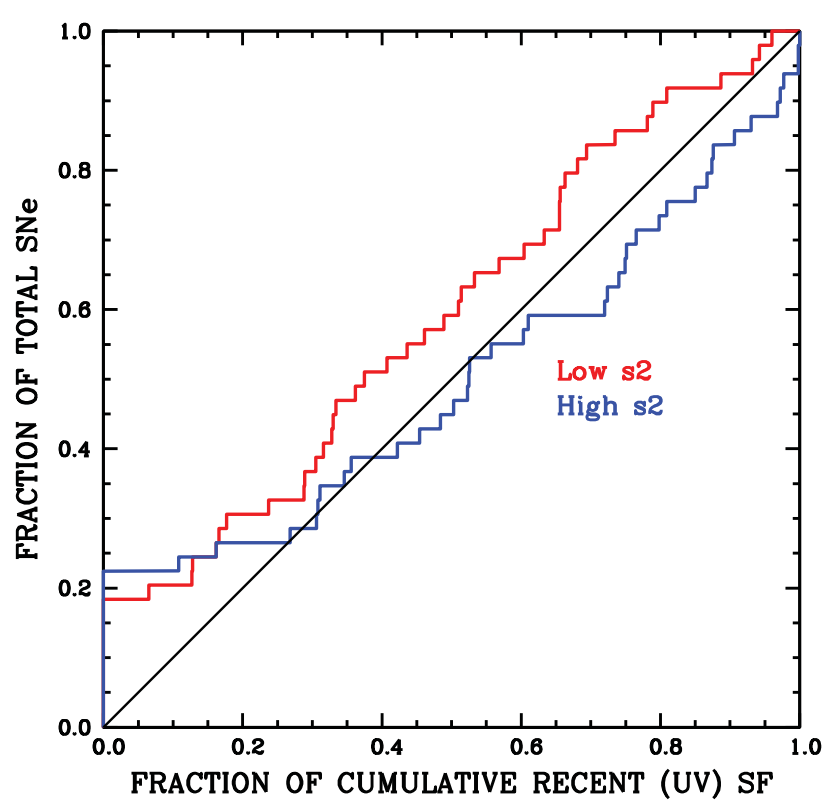

Figure 22. Near-UV NCR distributions for SNe II when these SNe are split by their light-curve decline rate parameter: $s_{2}$. The latter values are taken from Anderson et al. (2014b).

rameters were defined then measured. A full analysis of all parameters and all possible environment measurements will be left for future publications. Here, we present a few interesting figures using the analysis methods outlined in previous sections. In Anderson et al. (2014b), a parameter ' $s_{2}$ ' was defined to give the decline rate of SNe II during their 'plateau' phases (which are not necessarily flat, see that publication for in-depth discussion). Using such a parameter allows one to move away from simple 'IIP' or 'IIL' classifications. In Figure 22, we present near-UV NCR distributions for SNe II when the sample is split into low and high $s_{2}$ populations (slow and fast decliners). One can see that the faster 


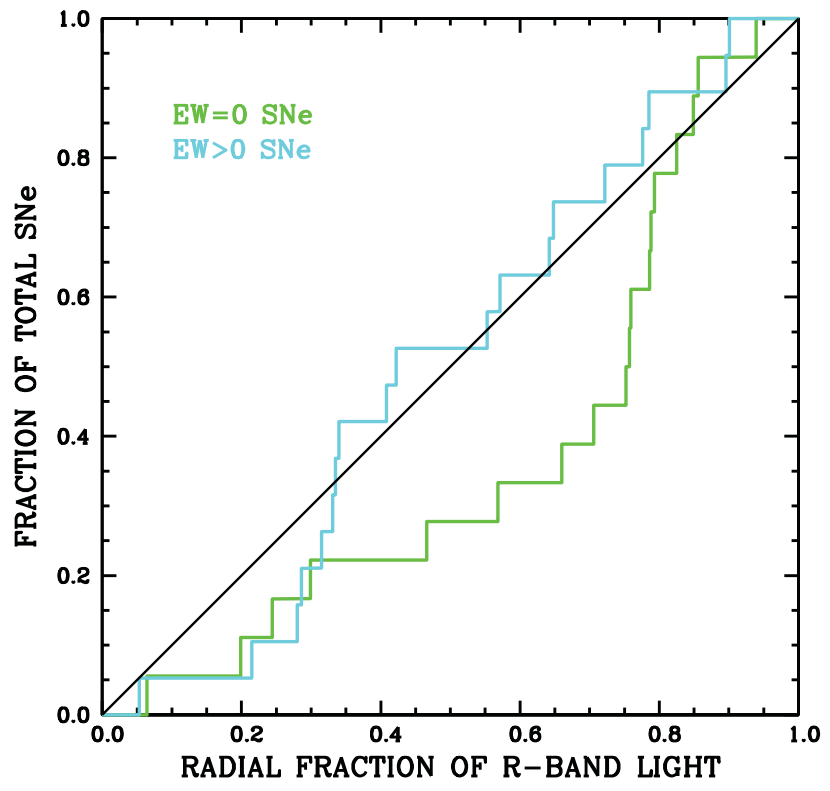

Figure 23. $\mathrm{Fr}_{R}$ distributions for $\mathrm{SNe}$ II with and without narrow sodium absorption detections within their spectra.

decliners show a higher degree of association to the recent $\mathrm{SF}$ within galaxies.

Host galaxy SN extinction is a key parameter in obtaining intrinsic $\mathrm{SN}$ brightnesses, however especially in the case of SNe II, a clear method for estimating this parameter is not forthcoming (see e.g. discussion in Faran et al. 2014b). One measurement which is often used to define the presence of host galaxy $A_{v}$ and its amplitude is the EW of narrow sodium lines within spectra. However, the accuracy of this measurement is debated (see discussion in Phillips et al. 2013 as applied to SNe Ia). It can be thus enlightening to see how sodium absorption within $\mathrm{SNe}$ II spectra changes as a function of environment. In Figure 23, $F r_{R}$ distributions are plotted for SNe II which do/do not show the presence of narrow sodium absorption within their spectra. One can see that those events with detections occur at smaller galactocentric radii than those without. This is to be expected as within the more central regions within galaxies a higher degree of ISM extinction is expected.

Finally, in Figure 24 we show SN II H II region metallicities, when the sample is split by the Anderson et al. (2014b) parameter OPTd which is a measure of the optically thick phase duration of the light-curve and measures the time between explosion and the end of the 'plateau' stage. It is generally assumed that the length of this light-curve phase is related to the mass of the hydrogen envelope at the epoch of explosion. While the statistics are small, it is observed that $\mathrm{SNe}$ with longer OPTd phases appear to explode in higher oxygen abundance regions than their shorter OPTd counterparts.

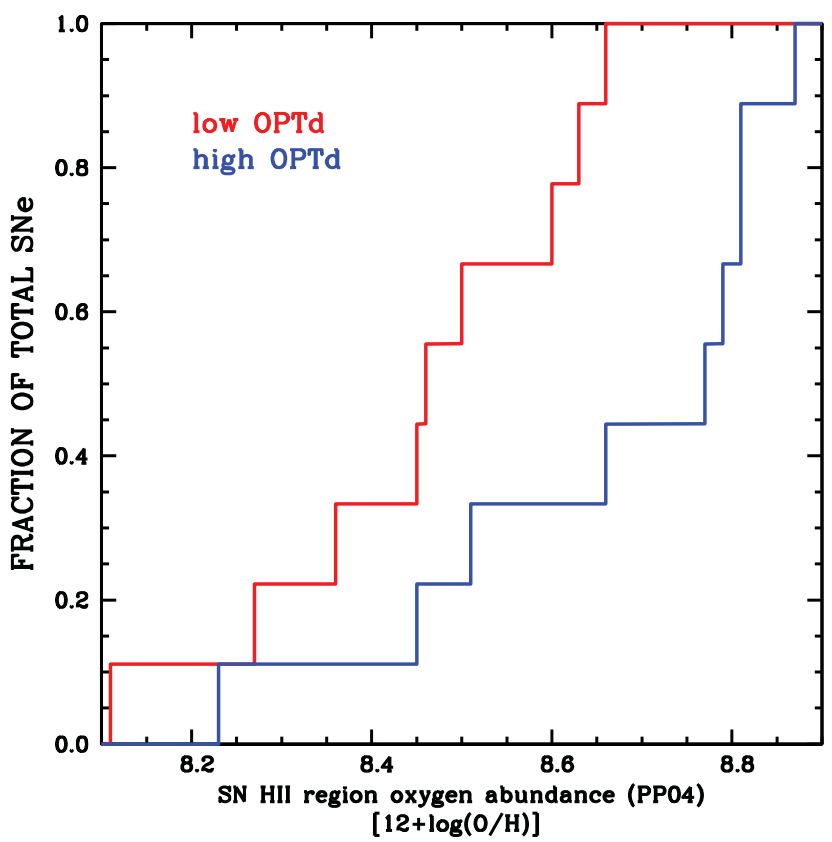

Figure 24. H II region metallicities for SNe II when split into equal samples of OPTd, the optically thick phase durations of their light-curves. The latter values are taken from Anderson et al. (2014b).

\section{DISCUSSION}

In the above, we have reviewed current results of SN environment studies, and shown that significant differences exist in the properties of the explosion sites of different SNe. These differences need to be explained by any proposed progenitortransient mapping scenario. Environmental results provide constraints on SN population progenitor properties such as lifetime, mass and metallicity, where these also need to be tied to the high probability that a significant fraction of $\mathrm{CC}$ $\mathrm{SNe}$ arise from binary systems. In this section, we outline how environmental results can be used to infer progenitor properties, and also discuss issues with the interpretation of the above results. Before moving to that discussion, we note an important point with regards to environmental studies. These investigations are statistical in nature, in the sense that any single measurement, be it association to an $\mathrm{H}$ II region, an emission line metallicity measurement, an environment colour etc, does not necessarily imply a strong progenitor constraint on each individual SN. This is the case because of limiting factors such as superposition of foreground or background stellar populations onto that of the specific SN, line of sight extinction which may mask specific environment properties, and progenitor velocities (from e.g. kicks due to previous SN explosions within a binary system). However, statistical studies allow one to overcome these limitations as one can look at how different $\mathrm{SN}$ populations compare in terms of overall environmental properties. Hence, if an SN population has a mean e.g. metallicity higher than another, then this constrains the overall population of the former to have higher progenitor metallicities than the latter. This may 
seem like a pedantic point, but it is important to keep in mind when discussing statistical constraints together with individual transient properties.

\subsection{Environmental constraints on $\mathrm{SN}$ progenitor mass}

We have shown above that SN types have an increasing degree of association to host galaxy SF, starting from SNe Ia, through SNe II, SNe Ib and finally SNe Ic (an observational result which has also been observed with various degrees of statistical significance by Kelly et al. 2008, Crowther 2013, Kangas et al. 2013, and Galbany et al. 2014). A major assumption in tying this to progenitor constraints is that a larger degree of association of any given SN type to especially the youngest ongoing $\mathrm{SF}$ traced by $\mathrm{H} \alpha$ emission, implies shorter stellar lifetimes and hence more massive progenitors.

The vast majority of stars form in clusters (Lada \& Lada 2003, although see Bressert et al. 2010), while massive stars are generally born in clustered environments (Portegies Zwart, McMillan, \& Gieles 2010, but see Wright et al. 2014). Massive young stars provide the ionising flux to create $\mathrm{H}$ II regions. As time passes, the most massive stars within a cluster explode as the first set of SNe. When there are no surviving stars of sufficient mass, then an $\mathrm{H}$ II region will cease to exist. In addition as time passes clusters will tend to dissipate, and hence longer lived progenitors will be less likely to be associated with their parent cluster. The passage of time also enables progenitors to move away from their parent cluster if they have some peculiar velocity with respect to the cluster. As time passes, gas will also be removed from a cluster due to the winds of the most massive stars, together with the first set of SN explosions. A full discussion of these processes (and others) can be found in Anderson et al. (2012). Following this, the simplest interpretation of Figure 1 is that $\mathrm{SNe}$ Ic have shorter lifetimes than $\mathrm{SNe} \mathrm{Ib}$, which in turn have shorter (but quite similar) lifetimes as the progenitors of SNe II. Hence, SNe Ic have higher progenitor masses than $\mathrm{SNe} \mathrm{Ib}$, which have higher masses than SNe II.

It is important to note here, our argument for higher mass progenitors for SN Ic as compared to SN Ib and SN II does not necessarily constrain the former progenitors to be single stars. It simply states than on average SN Ic have shorter lifetimes and more massive progenitors than $\mathrm{SN} \mathrm{Ib}$ and $\mathrm{SN}$ II, irrespective of the progenitor scenario. Indeed, this interpretation gains weight from the age constraints published by Galbany et al. (2014), and shown in Figure 14 (we also note that the higher resolution investigation of Kuncarayakti et al. 2013a gave qualitatively similar results, however conclusions are less clear due to small number statistics). In the case of $\mathrm{SNe} \mathrm{Ib}$, the fact that they show a very similar degree of association to ongoing SF as SNe II, indicates that the $\mathrm{SN} \mathrm{Ib}$ progenitor population is dominated by binary systems. This is because if $\mathrm{SNe}$ II and $\mathrm{SNe} \mathrm{Ib}$ arise from similar mass progenitors (implied from their NCR distributions), then single star mass-loss processes are insufficient to remove the entire hydrogen envelope. Mass transfer between binary companions can explain this result, and the importance of binary systems in understanding CC SN progenitors is discussed in detail below.

Crowther (2013) analysed very nearby CC SN positions with respect to $\mathrm{H}$ II regions, and in particular discussed the ages of both individual isolated regions, and large giant nebulae. This author found that $\mathrm{SNe} \mathrm{Ibc}$ were more associated to star-forming regions than $\mathrm{SNe}$ II, consistent with what has been outlined above. Indeed, Crowther also concluded that this implied higher mass progenitors for SNe Ibc than SNe II (we re-iterate that this does not necessarily differentiate between single and binary progenitor scenarios). An important difference between that work and the NCR pixel statistics above is the lack of statistics which did not allow Crowther to separate $\mathrm{SNe} \mathrm{Ib}$ from $\mathrm{SNe}$ Ic. When one obtains sufficient statistics to enable this separation, there is a clear difference between $\mathrm{SNe} \mathrm{Ib}$ and $\mathrm{SNe}$ Ic in terms of their preference for exploding near to $\mathrm{H}$ II regions, with the latter showing a much higher association. Indeed, this is a key observational result which needs to be explained through progenitor understanding. The current simplest interpretation is that $\mathrm{SNe}$ Ic arise from higher progenitor masses than $\mathrm{SNe} \mathrm{Ib}$.

One of the most intriguing results to arise from SN environmental studies are the properties of the explosion sites of SNe IIn. These explosions have generally been proposed to arise from very massive progenitors, possibly LBVs (see e.g. Smith 2008), and there is support for this view from e.g. the progenitor detection of SN 2005gl (Gal-Yam \& Leonard 2009). However, it has been shown that the environments of SNe IIn (and indeed their close cousins the SN 'impostors') are somewhat inconsistent with having very high mass progenitors (see above results and those in Anderson et al. 2012, Kelly \& Kirshner 2012, and Habergham et al. 2014). Indeed, SNe IIn have very similar environments as SNe IIP. In Anderson et al. (2012) and Habergham et al. (2014), it has been argued that this implies that SN IIn progenitors therefore have similar mass progenitors to SNe IIP, i.e. that they arise from the lower end of the CC SN progenitor mass range. There is some support for this view from individual SNe IIn/impostor events. The transient SN 2008S was constrained to have a progenitor mass of $\sim 10 \mathrm{M}_{\odot}$, from Spitzer pre-explosion images (Prieto et al. 2008b). Smith (2013) argued that the Crab nebula is the result of an SN IIn arising from a low-mass progenitor which exploded through the electron capture SN mechanism, and suggested that other SNe IIn showing a plateau in their light-curves (SNe IIn-P) may arise from similar progenitors.

One of the issues with analysing statistical samples of SNe IIn and 'impostors' is their heterogeneous nature (see discussion in Habergham et al. 2014). It is highly probable that there are multiple channels to produce the observed interacting transient diversity (see e.g. samples published in Kiewe et al. 2012 and Taddia et al. 2013b). Further separation of samples into all distinct classes removes any kind 
of statistical significance of analysis, and is also hampered by a lack of well-observed events. Such analysis is warranted in the future as the number of well observed events increases. However, aside from these issues one can still look at the overall sample in a statistical sense, and as above, this implies that the majority of SNe IIn do not arise from the most massive stars.

Motivated by the above statistical results, Smith \& Tombleson (2015) investigated the environments of LBV stars within the Milky Way and both the LMC and Small Magellanic Clouds (SMC). Measuring the distance between LBV stars and the nearest $\mathrm{O}$ stars, these authors found LBVs to be situated in isolated environments, at significant distances from collections of $\mathrm{O}$ stars. Given that the general assumption is that LBV stars are an evolutionary phase which $\mathrm{O}$ stars will pass through before becoming WR stars, this is very surprising. Smith \& Tombleson argued that this must imply two things. (1) that LBV stars are the mass gainers within massive binary systems. This would mean that the measured masses for LBV stars are actually significantly higher than Zero Age Main Sequence (ZAMS) masses. And (2) that the higher mass companions within these massive binary systems explode first and hence produce a significant kick which displaces LBV stars from their parent cluster, hence appearing in apparent isolation.

The location of LBV stars is possibly constraining for the nature of both SNe IIn and SN 'impostors'. If these isolated LBV stars are then the progenitors of a significant fraction of observed SNe IIn and 'impostors', then Smith \& Tombleson (2015) argue that this could explain the relatively low association of these interacting transients with host galaxy SF, without implying such low progenitor masses as derived for SNe IIP. It is also argued that such binary processes will dilute any information that can be gained on progenitor masses from environment studies. However, while there may indeed be some dilution of association with $\mathrm{H}$ II regions if a large fraction of SN progenitors have significant velocities, there are several issues with such claims. First, for an LBV progenitor to obtain a kick velocity, an initial SN has to explode. Within the vast majority of massive binary systems, the most massive star will explode first as core evolution is independent of surface mass-loss processes. These first $\mathrm{SNe}$ are most likely to be of type Ic. Hence, in such a system the most massive explosion will be observed to be significantly associated with their host stellar population, while when the LBV finally evolves to explosion, it will be less associated because (a) it has received a velocity kick and will hence explode further away from the host $\mathrm{H}$ II region, however importantly (b) the latter will obviously explode after the initial $\mathrm{SN}$, and hence all previous arguments for an increased association to SF being equivalent to younger progenitor ages still apply. Therefore, the initial mass difference between progenitors within the system will still be traced by environment properties even in a binary velocity kick scenario. If these kicked LBVs were the progenitors of SNe IIn, then the latter would still have lower progenitor masses than the first $\mathrm{SNe}$ to explode, i.e. SNe Ic, as we have argued above. The conclusions of Smith \& Tombleson (2015) are intriguing and if true would have significant implications for stellar life and death. Future observations are needed to test the predictions of their scenario.

In conclusion, we believe that differences in the association of SNe to host galaxy SF indeed imply differences in mean progenitor ages and masses. Exact progenitor mass constraints are more difficult, especially when tied in with the complications of binaries above. Finally, we stress that differences in the association of SNe to SF need to be explained by any valid progenitor scenario. Evidence for binary progenitors for CC SNe is now outlined in more detail.

\subsubsection{Binary progenitors of CC SNe}

It is now firmly established that the majority of massive stars will have their evolution significantly affected before explosion because of the influence of a binary companion (Sana et al. 2012). The presence of a binary companion may not only affect the evolution of a star, but also affect its positional characteristics, i.e. its environment, after the initial star within the system explodes. Indeed, it has long been suggested that binary evolution can lead to a number of observed $\mathrm{SNe}$, especially for the stripped envelope types of Ibc, and IIb (e.g. Podsiadlowski, Joss, \& Hsu 1992).

Sana et al. (2012) found that $\sim 70 \%$ of massive O stars will lose mass through binary interaction (also see Sana et al. 2013). This being the case, it is impossible to ignore the effects of binary interactions on $\mathrm{SN}$ progenitors and subsequent SN diversity. CC SN rates also give strong evidence that at least a fraction of SNe Ibc must arise from binaries (Kobulnicky \& Fryer 2007; Smith et al. 2011). Smith et al. (2011) analysed the volumetric rates of CC SNe from the Lick Observatory Supernova Search (LOSS), and compared these to the expected number of SNe within different progenitor mass ranges assuming a standard IMF. The main conclusion was that single star scenarios cannot explain the observed rate of $\mathrm{SNe}$ Ibc. These authors created various progenitor scenarios, and favoured a hybrid scheme where the majority of SNe Ibc, in particular the $\mathrm{SNe} \mathrm{IIb}$ and $\mathrm{SNe} \mathrm{Ib}$, arise from binary progenitors, but some SNe Ic are still produced by single stars. This scenario qualitatively agrees with the above findings with respect to environments. Indeed, Smith et al. (2011) predicted that following this scenario $\mathrm{SNe}$ Ic should have environments which are more consistent with higher masses and higher metallicities than $\mathrm{SNe}$ II and $\mathrm{SNe} \mathrm{Ib}$, as is concluded in Sections 3.1 and 3.3. In addition, Eldridge, Izzard, $\&$ Tout (2008) showed that a mix of binary and single star synthesised progenitor populations most accurately map the observed CC SN ratios and their dependence on metallicity.

There is also evidence for binary progenitors through analysis of individual events. Bersten et al. (2014) modelled the early light-curve of the SN Ib iPTF13bvn, and constrained its progenitor to be relatively low mass, which would only be possible in a binary scenario. In the case 
of $\mathrm{SNe} \mathrm{IIb}$, there are several progenitor detections which indicate a binary progenitor scenario. After SN 1987A, SN 1993J was the second $\mathrm{SN}$ to have a progenitor detection (Aldering, Humphreys, \& Richmond 1994). A companion star was later observed, hence confirming the binary nature of this explosion (Maund et al. 2004; Maund \& Smartt 2009). A progenitor detection was also made for SN 2011dh (Van Dyk et al. 2011; Maund et al. 2011). In this case, there was some debate as to whether the detection was the actual progenitor, and also as to its single or binary nature. Bersten et al. (2012) argued for a low-mass progenitor star, consistent with that detected in pre-explosion images, which constrained the progenitor to be a binary system. Folatelli et al. (2014) have claimed a possible detection of the companion star to SN 2011dh in late time Hubble Space Telescope images, which would be consistent with the above model predictions of Bersten et al. (2012). In the case of $\mathrm{SNe}$ Ic, we still await a sufficiently nearby and well-studied explosion to enable such constraints.

While SNe II now have a significant number of direct progenitor detections, there is a lack of pre-explosion SN Ibc progenitor detections, where only limits have been possible on progenitor luminosities, as shown by Eldridge et al. (2013). These authors used this evidence, together with the above constraints on $\mathrm{SN}$ relative rates to argue for binary progenitors for the majority of $\mathrm{SNe} \mathrm{Ibc}$, and presented binary evolution models to account for observational constraints.

The discussion in this section clearly shows that there is significant evidence that binary systems account for at least a fraction of CC SN diversity. Hence, these results have to be taken into account when discussing environmental results. However, while the presence and influence of binary companions may significantly affect the nature of the transients themselves, environmental results can still provide strong constraints on the nature of progenitors, and further constraints on the fraction of progenitors that arise from single or binary scenarios.

\subsection{Radial distributions of CC SNe}

The long-standing assumption that the centralisation of $\mathrm{SNe}$ Ibc with respect to $\mathrm{SNe}$ II within galaxies is due to a progenitor metallicity effect (see e.g. van den Bergh 1997; Hakobyan et al. 2009; Anderson \& James 2009), can no longer be deemed valid. We have shown that this result is dominated by $\mathrm{SNe}$ within disturbed galaxies where any metallicity gradients which precede such an assumption will be much less prevalent, or even non-existent. Hence, other explanations must be explored. Such discussion has been detailed in Habergham et al. (2010), Anderson et al. (2011), and Habergham et al. (2012). We summarise the main points here.

In the central parts of disturbed and interacting systems, starbursts of violent SF are often frequently found to occur (see e.g. Larson \& Tinsley 1978; Joseph \& Wright 1985), leading to a high turnover of massive stellar birth and death.
One possibility to explain $\mathrm{SNe}$ Ibc centralisation could thus be that we are simply observing systems at an epoch when the the most massive stars, i.e. the SNe Ibc, are exploding following a burst of SF. However, this would require extreme fine tuning in the sense that the difference in lifetimes between SNe II and SNe Ibc (in any given progenitor scenario) are at most several 10s of Myrs. Meanwhile galaxy mergers/interactions have timescales of several 100 Myrs (see the results of models of merging galaxies in Mihos \& Hernquist 1996). Hence, it is not clear how the radial distribution of CC SNe within disturbed galaxies can be explained though progenitor age effects. While this could be the case for any one given galaxy (e.g. see the SN distribution in Arp 299, Anderson et al. 2011), it is implausible that we are observing the rest of our disturbed sample (such as that displayed in Figure 4, where it seems obvious that these galaxies display a wide range of starburst ages) when the dominant the age of massive stars is only a few Myrs. Again, we encourage the reader to browse the content of Habergham et al. (2012) for further discussion of this issue, and some more quantitative arguments.

Another possibility is that the binary fraction of massive stars changes with environment. It is quite possible that the SF within the central parts of disturbed systems is dissimilar to that found in 'normal' star-forming regions in galaxy disks ${ }^{4}$. However, it is not clear in which direction this will go. Denser SF may indeed lead to a higher fraction of binaries, however those binaries may be more easily disturbed by nearby stellar systems. It is possible that some binary systems are disrupted, but it is also possible that those remaining binaries become more compact. The latter become close-binaries which are more likely to influence stars before explosion, as these hard binaries will lead to mass exchange on the main sequence (e.g. de Mink et al. 2014), and thus a larger fraction of progenitors could explode as SNe Ibc. These issues are not clear. However, within this discussion there is an important point which is usually ignored. If a binary system is to produce a SN Ibc, where the components have insufficient mass to lose their envelopes through single star processes, then this implies that the companion has gained hydrogen-rich material (or at the very least not lost a significant amount of its own envelope). Hence, the second star to explode within the system will most likely be hydrogen-rich and hence an SN II. Thus, even in a stellar population where all SNe were produced by progenitors which had undergone binary mass transfer, the lower mass companion is most likely to explode with a significant fraction of its hydrogen envelope intact. This would imply that the ratio of SNe II to SNe Ibc in such hypothetical environments would be close to equal. We have shown above that in some environments this is clearly not the case (in the central parts of the extremely disturbed galaxies in the above sample three times more $\mathrm{SNe}$ Ibc than $\mathrm{SNe}$ II

\footnotetext{
${ }^{4}$ We also note that it is possible that the binary fraction is higher within the central parts of bright $\mathrm{H}$ II regions, and could be a factor in the interpretation of the results presented in Section 3.1.
} 
have been observed). Hence, while we cannot currently disprove that SF in the central parts of these galaxies produces a higher fraction of CC SNe from binaries, it is not clear how this would explain the above results. Nevertheless, studies of the fraction of massive stars within binaries as a function of environment will be an interesting direction for such work in the future.

It is also possible that interaction driven SF in the central regions of galaxies produces a different distribution of stars than those within 'normal' SF regions. One way to explain the centralisation of $\mathrm{SNe} \mathrm{Ibc}$ is to assume that these $\mathrm{SNe}$ arise from more massive stars than $\mathrm{SNe}$ II (for which we believe there is significant evidence above), and then that the IMF is biased towards higher mass progenitors in these regions. Such a claim is indeed controversial, as there is significant doubt as to whether the IMF varies with environment (see Bastian, Covey, \& Meyer 2010 for a review). However, there are predictions, such as those from Klessen, Spaans, \& Jappsen (2007), who suggest that in extreme SF environments the IMF may indeed be biased towards highmass stars, with a cut off at lower masses. We do not go into more detail on this topic here, however we believe that such a possibility needs to be considered when discussing $\mathrm{SN}$ environment properties.

\subsection{Progenitor metallicity constraints}

Single star stellar evolution models predict that there should be a significant dependence of the SN II/SN Ibc ratio on progenitor metallicity (e.g. Heger et al. 2003; Eldridge \& Tout 2004; Georgy et al. 2009). Binary population models (such as Eldridge et al. 2008) predict a much less significant trend with metallicity, as the mass-loss is dominated by mass transfer in place of metallicity-dependent winds. Studies of the global properties of SN host galaxies have observed that the SN II to SN Ibc ratio is dependent on: galaxy luminosity (Prantzos \& Boissier 2003; Boissier \& Prantzos 2009; Arcavi et al. 2010), which can be used as a proxy for galaxy metallicity; and galaxy metallicity (Prieto, Stanek, \& Beacom 2008a). These studies have argued that these results imply a significant difference in environment and thus progenitor metallicities of SNe II and SNe Ibc. However, above we have already shown that the interpretation of the radial distributions of CC SNe cannot be fully explained through progenitor metallicity effects. In Section 3.3 of this review, we have shown that there are only small differences between CC SN host $\mathrm{H}$ II region metallicities between $\mathrm{SNe}$ II and SNe Ibc. This is then in conflict with the predictions of single star progenitor scenarios, as there is only a suggestion that the SN II to SN Ibc rate increases with decreasing oxygen abundance, and only in the lowest $\mathrm{H}$ II region bin. These results appear to be strong additional evidence that a significant fraction of SN Ibc progenitors arise from binary systems.

It is also interesting to note that while any differences are statistically marginal, of all the main CC SN types SN Ic do explode in the regions with the highest oxygen abundances. In Figure 11, there is some suggestion that the $\mathrm{SN}$ Ib to SN Ic rate increases with decreasing metallicity. It was also shown in Section 3.2 that in undisturbed systems, where metallicity gradients are measured to be more prevalent, $\mathrm{SNe}$ Ic are indeed found to explode more centrally than $\mathrm{SNe} \mathrm{Ib}$, lending further weight to a progenitor metallicity difference between $\mathrm{SNe} \mathrm{Ib}$ and SNe Ic. Hence, following the discussion above in Section 4.1, environmental properties appear to constrain $\mathrm{SNe} \mathrm{Ib}$ to have quite similar progenitor properties in terms of mass and metallicity to SNe II, while SNe Ic are constrained to have higher mass and metallicity ${ }^{5}$ progenitors than both the former. If correct, this hypothesis would imply that the majority of $\mathrm{SNe} \mathrm{Ib}$ arise from binary progenitors, while we suggest that at least a fraction (if not all) SNe Ic arise from single star progenitors.

\subsection{Progenitor constraints in the wider context}

In this section, we briefly summarise other $\mathrm{SN}$ progenitor analyses in the context of environment work. Direct progenitor detections give the most stringent constraints on the origin of SNe. SNe IIP are the only type to have a significant number of progenitor detections, where their initial masses have been constrained to be between 8 and $\sim 20 \mathrm{M}_{\odot}$ (see Smartt et al. 2009 for a review, and Maund et al. 2013 and Fraser et al. 2014 for recent examples). This is consistent with environmental constraints that SNe IIP arise from the lower end of the CC SN progenitor mass range, and their accurate tracing of host galaxy near-UV emission (Figure 2). There are suggestions from environmental studies that SNe IIL have higher masses than SNe IIP (see e.g. Figure 22). Only a couple of progenitor detections of SNe IIL exist, however they do appear to be higher in mass than SNe IIP (Elias-Rosa et al. 2010; Fraser et al. 2010; Elias-Rosa et al. 2011). The lack of detections of SN Ibc progenitors, as shown in Eldridge et al. (2013), has been used to argue for binary progenitors for these $\mathrm{SNe}$. However, we stress that any such study should separate $\mathrm{SNe} \mathrm{Ib}$ from $\mathrm{SNe}$ Ic given their distinct environments.

Leloudas et al. (2010) investigated the spatial distribution of WR stars within galaxies. Using a similar technique to the pixel statistics methods outlined above, these authors found that WR stars are found to be located in similar environments as $\mathrm{SNe}$ Ibc, thus supporting the possibility that some of the progenitors of the latter are high-mass single stars. It was also found that SNe Ic showed environmental properties more similar to WC stars, and $\mathrm{SNe}$ Ib to WN stars, consistent with the scenario that SNe Ic are explosions of stars that have been more heavily stripped of their envelopes. It may be interesting to further these studies for other stellar types, such as red and blue supergiants, and look in more detail at the association of different stars to more direct tracers of $\mathrm{SF}$, such as $\mathrm{H} \alpha$. We also recall the investigation of Smith

\footnotetext{
${ }^{5}$ Although we note that the subclass of these events, SNe Ic BL, appear to favour lower metallicity environments. See e.g. Modjaz et al. (2011).
} 
\& Tombleson (2015), which sheds light on the spatial distribution of different massive stars (and hence possible SN progenitors) with respect to each other.

There has also been work constraining SN progenitors through analysis of resolved stellar populations within very nearby galaxies (Gogarten et al. 2009a; Murphy et al. 2011; Williams et al. 2014; Jennings et al. 2014). These studies estimate the age of resolved parent stellar populations (on scales of $\sim 50 \mathrm{pc}$ ) and translate these to progenitor masses. As we observe additional $\mathrm{SNe}$ in the very nearby Universe, and as the next generation of extremely large telescopes come on line, the possibilities of these studies will only increase.

Lyman et al. (2014a) studied the properties of a sample of 38 stripped envelope $\mathrm{SNe}$ ( $\mathrm{SNe} \mathrm{IIb}$, Ib, Ic, Ic-BL). These authors concluded that derived ejecta masses imply that lowmass binary progenitors dominate the progenitor channel of these SNe. Interestingly, they did find that $\mathrm{SNe}$ Ic have higher ejecta masses than $\mathrm{SNe} \mathrm{Ib}$, perhaps implying higher mass progenitors for the latter, as suggested by environmental results above.

\subsection{Selection effects of environment observations and SN samples}

There are now a large number of SN environmental studies, as has been discussed throughout this review. There are many selection biases that may affect different studies, both in the nature of the environment studies, and in the nature of the SN samples themselves. Here, we briefly summarise these, but we refer the reader to the literature cited throughout this review for more complete discussions.

Exact objective SN typing is still an issue today when one has insufficient light-curve or spectral information. Indeed, many $\mathrm{SNe}$ are simply classified spectroscopically and then are not followed any further. This problem may be more important for analyses based on old literature $\mathrm{SNe}$, when less data were obtained, and less was known about the diversity of $\mathrm{SNe}$ and its physical origin. However, it is not clear why this problem should bias environment results in any particular direction.

In Section 3.3, we combined the measurements of many different host $\mathrm{H}$ II region oxygen abundance studies. While these studies were all of resolved galaxies, the redshift range of their samples is quite different, with some very nearby studies such as Kuncarayakti et al. (2013a, 2013b) combined with more distant samples. As one goes to larger distance, the reduction in spatial resolution means that one's measurement is over a much larger environment. Other than diluting the information of the exact parent stellar population, it is unclear how this will affect the results of environment studies. One issue that is often cited is that if there is any significant difference in the luminosities of different $\mathrm{SNe}$ which are being compared, then this will result in a bias against the detection of one SN type over the other against e.g. bright $\mathrm{H}$ II regions, the brighter centres of host galaxies, or within regions of higher host galaxy extinction. While SNe Ia are significantly brighter than $\mathrm{CC} \mathrm{SNe}$, there is actually little difference between the peak absolute magnitudes of SNe II and $\mathrm{SNe} \mathrm{Ibc}$, as shown by SNe discovered within the LOSS survey ( $\mathrm{Li}$ et al. 2011). Hence, there is little evidence for any selection bias that could explain the preference of SNe Ibc for exploding in both more central regions of host galaxies, and within bright $H$ II regions.

The exact make-up of host galaxy samples is also often discussed in terms of a bias between different studies. While traditional SN searches have concentrated on targeting massive, bright galaxies, there now exist a number of untargeted searches which scan the sky searching for $\mathrm{SNe}$, and find many more $\mathrm{SNe}$ in low surface brightness galaxies than the former. Undertaking environment studies in these different samples will affect the relative numbers of studied SNe and may also change the nature of those SN populations. However, it is interesting to note (as already stated above) that Stoll et al. (2013) found that an SN II sample from an untargeted search had a very similar $\mathrm{H}$ II region oxygen abundance distribution as that that derived from a sample of literature $\mathrm{SNe}$ discovered by targeted searches.

All of the above issues need to be taken into account when studying SN environments, and future studies will strive to reduce any systematic biases in their samples, to obtain the most robust progenitor and transient constraints.

\section{CURRENT AND FUTURE PROSPECTS}

SN environment studies are still in their infancy, and current/future instrumentation promises to further open the field for new results and analysis. There are two specific avenues which we believe will be pursued by environment studies to significantly increase insight on SN progenitors. The first involves the actual transients. This advancement was introduced in Section 3.7, and involves having large samples of well-characterised SNe. From these samples, one can then tie specific environment properties to specific SN features which may be more physical in nature than simple type classifications. The second involves new observations enabled by current and future instrumentation, and specifically the advantages gained for environment studies by using IFU observations. We now discuss this in more detail, and show some preliminary analysis in that direction.

\subsection{New types of environment observations}

We have already commented on the central importance of wide-field IFUs for future studies of SN host galaxies. This enables simultaneous spatial and spectral information over the entire extent of host galaxies. Indeed, the usefulness of IFU observations for SN host environment studies has already been demonstrated by e.g. Stanishev et al. (2012), Rigault et al. (2013), Kuncarayakti et al. (2013a, 2013b) and Galbany et al. (2014).

MUSE mounted on the VLT telescope promises to significantly enhance the capabilities of IFU instrumentation 


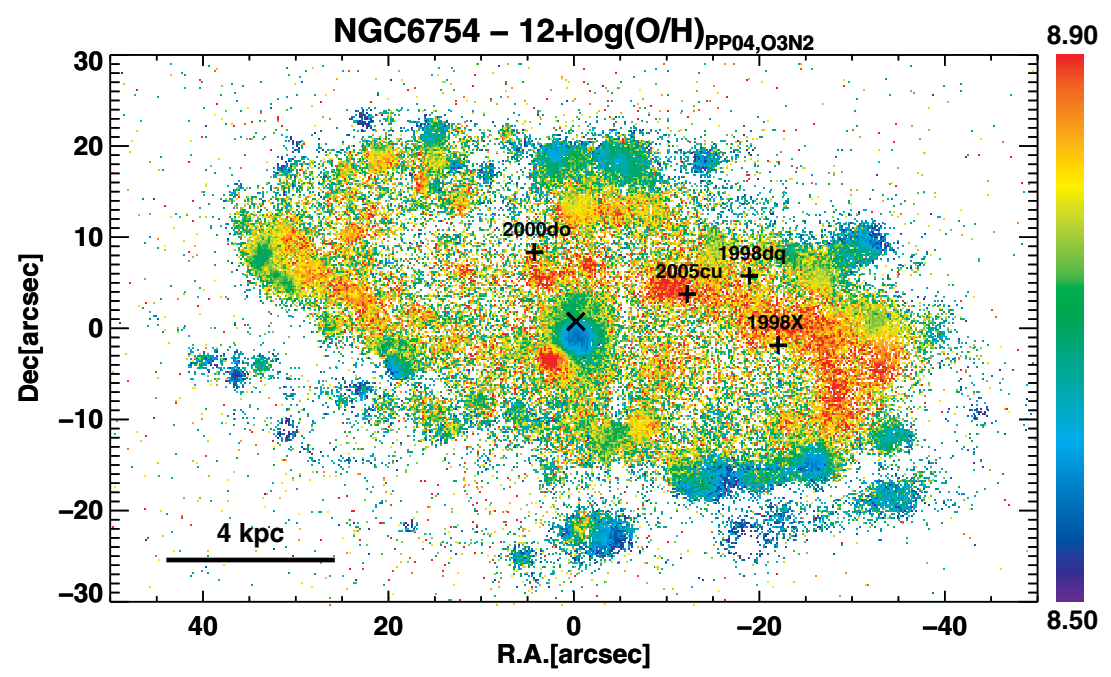

Figure 25. PP04 O3N2 metallicity map of NGC6754, with SN explosion sites indicated.

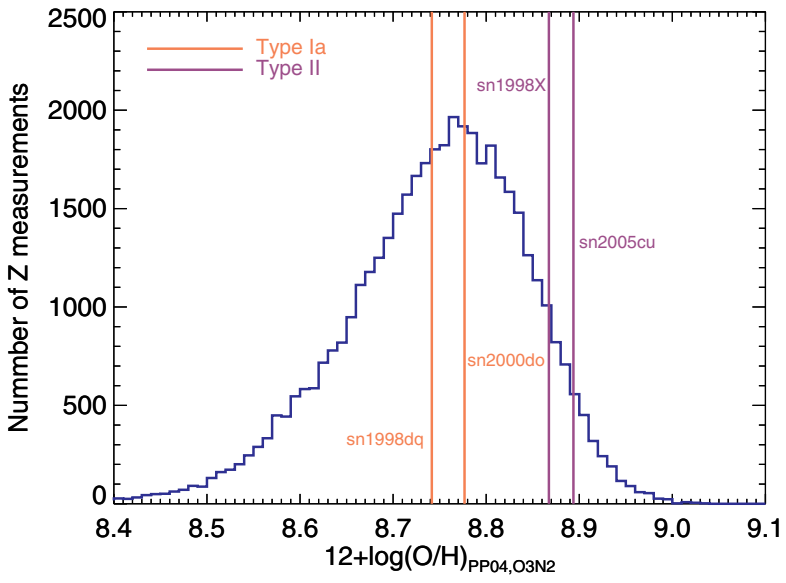

Figure 26. Histogram of PP04 O3N2 metallicity measurements of NGC 6754 , with SN environment metallicity positions indicated.

for environment studies, having a FOV of $1 \operatorname{arcmin} \times 1$ arcmin, extremely good spatial resolution $(0.2$ arcsec spaxel size), and the power of being attached to an 8-m class telescope. We recently obtained Science Verification data (P.I. Galbany), to observe the galaxy NGC 6754 which has been host to four SNe. A full analysis of that galaxy and other data taken during $\mathrm{SV}$ in terms of SN environmental parameters will be presented in L. Galbany et al. (in preparation). Here, we show a glimpse of the data obtained and show what can be achieved through such analysis. (Note, an analysis of the H II region population of NGC 6754 using these data has been presented in Sánchez et al. 2015.) In Figure 25, we present the spatial metallicity map of NGC 6754 on the PP04 O3N2 scale, with SN positions indicated. In Figure 26, a histogram of all measured metallicities is shown, with values estimated at exact $\mathrm{SN}$ sites indicated. These figures show the power of these data as compared to previous work, much of which was discussed above, where in the latter either spatial informa- tion of a specific emission line (e.g. $\mathrm{H} \alpha$ ) has been analysed, or spectral information but in one small-scale region. With current state-of-the-art instrumentation such as MUSE, one can obtain all these parameters with one single telescope pointing.

Additional instruments are also coming online to further these IFU possibilities, such as SAMI (Bryant et al. 2012), and MANGA (Law \& MaNGA Team 2014). WiFeS (Dopita et al. 2010; Childress et al. 2014a) is another instrument which is being used for these types of studies. These multiIFU instruments will observe thousands of galaxies. While $\mathrm{SN}$ environment science may not initially be the key science drivers, when one observes such quantities of galaxies, then one naturally obtains statistically significant samples of SN host galaxies, which can be used for environment studies. Using these instruments, one can essentially analyse host galaxies/environments in the manner described in Sections 2.1-2.3 simultaneously, i.e. one can extract any emission line (obviously within the constraints of any given instrument), or broad-band 'colour' and proceed with 'spaxel statistics'. This will enable environment parameters such as metallicity to not only be compared between SN types, but also between $\mathrm{SN}$ environments and all other environments within their host galaxy (this in essence is what is done above for the $\mathrm{H} \alpha$ pixel statistics). This is what is shown in Figure 26: a histogram of all host galaxy environment metallicity measurements. In this particular galaxy, it is observed that the SNe Ia explode within the peak of the metallicity distribution, while the SNe II appear to explode in regions of higher oxygen abundance. We note here that this analysis is very preliminary, and is shown here to present future possibilities. Published values may change once a more thorough analysis is achieved.

While large-scale statistical environment analysis will hugely benefit with the advent of wide-field IFU instrumentation, it is also interesting to attempt to observe environments with the best spatial resolution in order to observe individual progenitor parent stellar clusters. In order to obtain the best 


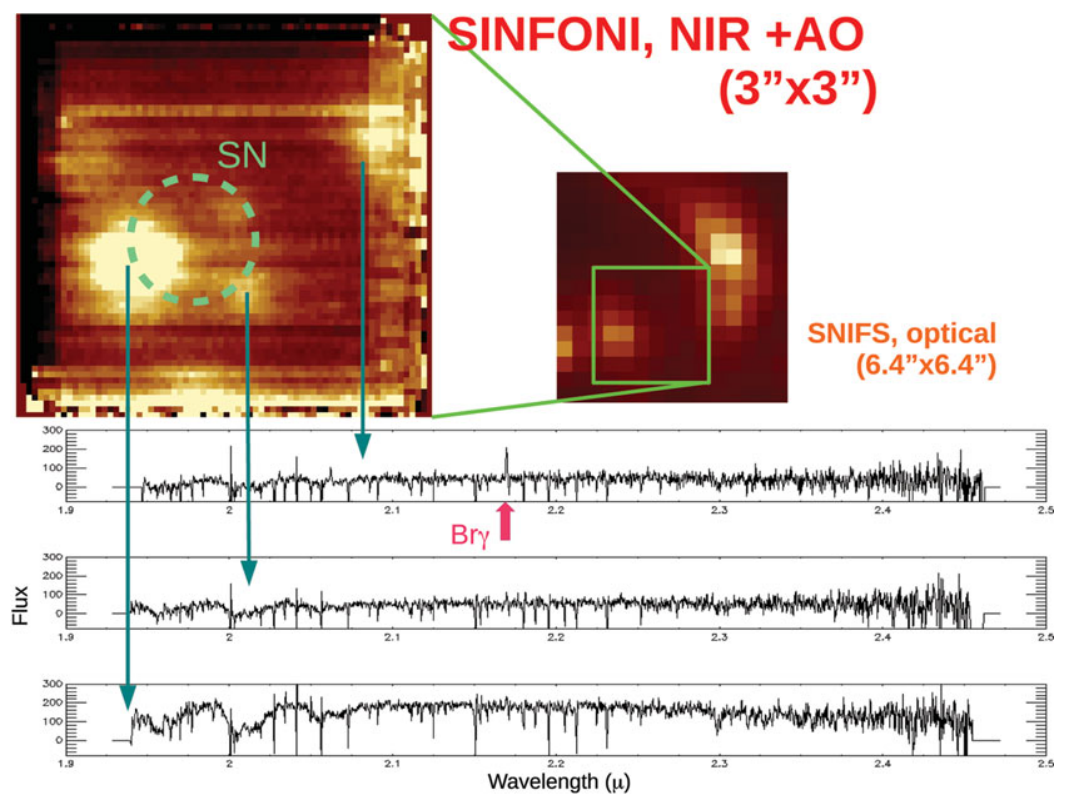

Figure 27. Example of the advantages of moving to the near-IR where one can obtain much higher spatial resolution due to adaptive optics corrections. A comparison is made between optical IFU (SNIFS) observations, and those obtained with SINFONI of the same SN environment. Also shown are the extracted near-IR spectra, where the Br-gamma emission line is indicated. (A similar figure is also shown in Kuncarayakti et al. 2014)

spatial resolution, adaptive optics (AO) helps to overcome the limitations of atmospheric conditions (i.e. seeing) during an observation. In this fashion, we have obtained data of a handful of SN environments with SINFONI (Eisenhauer et al. 2003), a near-IR IFU instrument. In Figure 27, we present an example of the gain in spatial resolution using near-IR AO observations, where SINFONI observations are compared to those of an optical IFU of the same environment. This shows that what appears to be a single cluster in an optical observation is resolved into several clusters in SINFONI observations. In the near-IR, one can use the presence or absence of Br-gamma emission to constrain the age of the environment/parent cluster (more details will be presented in H. Kuncarayakti et al. in preparation). Better spatial resolution also enables us to alleviate SF history issues.

The above examples show how the field of environments is set to evolve over the coming years. In addition, one assumes that investigators will find other innovative ideas to use other instruments to study SN environments. In combination with growing databases of high-quality follow-up data, the coming years promise to be particularly fruitful for the environment and $\mathrm{SN}$ fields.

\section{SUMMARY AND CONCLUSIONS}

The field of SN environments is still relatively young, but is growing into an important area of investigation. Major differences in the environmental properties of different $\mathrm{SNe}$ exist, and any hypothesised progenitor scenario must be able to explain such trends.

In this manuscript, we have reviewed the current status of SN environment studies, and how these can be used to constrain SN progenitors. Differences in the environments of SNe II, SNe Ib and SNe Ic appear to imply that the progenitor mass and metallicity ranges of the first two are quite similar, with larger values for the latter. This is explained if the majority of $\mathrm{SNe} \mathrm{Ib}$ arise from binary systems; however, we note that environmental results still allow for a significant number of SNe Ic to arise from higher mass, and possibly single star progenitors. These conclusions are in qualitative agreement with contemporary studies of SNe. The environmental properties of SNe IIn test our understanding of these events and their progenitors, and it will be intriguing to see what further investigation brings to this debate. While studies of SN Ia environments are perhaps less constraining, there indeed exist interesting results.

SN environment studies are rapidly evolving with new instrumentation, and ever larger $\mathrm{SN}$ samples to study. Future years promise to be rich with new analysis, and further constraints on the large diversity of SNe.

\section{ACKNOWLEDGEMENTS}

In no particular order we thank: Mario Hamuy, Francisco Förster, Santiago González, Stephen Smartt, Nathan Smith, Maryam Modjaz, Gaston Folatelli, Melina Bersten, Mark Phillips, Max Stritzinger, Paul Crowther, Giuliano Pignata, 
Franz Bauer, Morgan Fraser, Joe Lyman for many fruitful discussions which contributed to the content of this review. Support for HK and LG is provided by the Ministry of Economy, Development, and Tourism's Millennium Science Initiative through grant IC120009, awarded to The Millennium Institute of Astrophysics, MAS. HK acknowledges support by CONICYT through FONDECYT grant 3140563, and LG through grant 3140566 .

\section{References}

Aldering, G., Humphreys, R. M., \& Richmond, M. 1994, AJ, 107, 662

Anderson, J. P., Covarrubias, R. A., James, P. A., Hamuy, M., \& Habergham, S. M. 2010, MNRAS, 407, 2660

Anderson, J. P., Habergham, S. M., \& James, P. A. 2011, MNRAS, 416, 567

Anderson, J. P., Habergham, S. M., James, P. A., \& Hamuy, M. 2012, MNRAS, 424, 1372

Anderson, J. P., \& James, P. A. 2008, MNRAS, 390, 1527

Anderson, J. P., \& James, P. A. 2009, MNRAS, 399, 559

Anderson, J. P., James, P. A., Förster, F., González-Gaitán, S., Habergham, S. M., Hamuy, M., \& Lyman, J. D. 2015, MNRAS, 448,732

Anderson, J. P., \& Soto, M. 2013, A\&A, 550, A69

Anderson, J. P., et al. 2014a, MNRAS, 441, 671

Anderson, J. P., et al. 2014b, ApJ, 786, 67

Arcavi, I., et al. 2010, ApJ, 721, 777

Arcavi, I., et al. 2012, ApJL, 756, L30

Arnett, W. D., Bahcall, J. N., Kirshner, R. P., \& Woosley, S. E. 1989, ARA\&A, 27, 629

Baade, W., \& Zwicky, F. 1934, PNAS, 20, 254

Bacon, R., et al. 2010, in Proc. SPIE Conf. Ser., Vol. 7735, Groundbased and Airborne Instrumentation for Astronomy III, ed. I. S. McLean, S. K. Ramsay, H. Takami (Bellingham, SPIE), 8

Barbon, R., Ciatti, F., \& Rosino, L. 1979, A\&A, 72, 287

Bartunov, O. S., Makarova, I. N., \& Tsvetkov, D. I. 1992, A\&A, 264,428

Bartunov, O. S., Tsvetkov, D. Y., \& Filimonova, I. V. 1994, PASP, 106, 1276

Bastian, N., Covey, K. R., \& Meyer, M. R. 2010, ARA\&A, 48, 339

Bersten, M. C., et al. 2012, ApJ, 757, 31

Bersten, M. C., et al. 2014, AJ, 148, 68

Bianco, F. B., et al. 2014, ApJS, 213, 19

Boissier, S., \& Prantzos, N. 2009, A\&A, 503, 137

Bressert, E., et al. 2010, MNRAS, 409, L54

Bryant, J. J., et al. 2012, in Proc. SPIE Conf. Ser., Vol. 8446, Ground-based and Airborne Instrumentation for Astronomy IV, ed. I. S. McLean, S. K. Ramsay, H. Takami (Bellingham, SPIE), 0

Chen, T.-W., et al. 2014, arXiv e-prints: 1409.8827

Childress, M. J., Vogt, F. P. A., Nielsen, J., \& Sharp, R. G. 2014a, Ap\&SS, 349, 617

Childress, M. J., Wolf, C., \& Zahid, H. J. 2014b, MNRAS, 445, 1898

Chugai, N. N., \& Danziger, I. J. 1994, MNRAS, 268, 173

Crowther, P. A. 2013, MNRAS, 428, 1927 de Mink, S. E., Sana, H., Langer, N., Izzard, R. G., \& Schneider, F. R. N. 2014, ApJ, 782, 7

Dopita, M., et al. 2010, Ap\&SS, 327, 245

Drout, M. R., et al. 2011, ApJ, 741, 97

Eisenhauer, F., et al. 2003, in Proc. SPIE Conf. Ser., Vol. 4841, Instrument Design and Performance for Optical/Infrared Groundbased Telescopes, ed. M. Iye \& A. F. M. Moorwood (Bellingham, SPIE), 1548

Eldridge, J. J., Fraser, M., Smartt, S. J., Maund, J. R., \& Crockett, R. M. 2013, MNRAS, 436, 774

Eldridge, J. J., Izzard, R. G., \& Tout, C. A. 2008, MNRAS, 384, 1109

Eldridge, J. J., \& Tout, C. A. 2004, MNRAS, 353, 87

Elias-Rosa, N., et al. 2010, ApJL, 714, L254

Elias-Rosa, N., et al. 2011, ApJ, 742, 6

Faran, T., et al. 2014a, MNRAS, 445, 554

Faran, T., et al. 2014b, MNRAS, 442, 844

Filippenko, A. V. 1997, ARA\&A, 35, 309

Filippenko, A. V., Matheson, T., \& Ho, L. C. 1993, ApJL, 415, L103

Folatelli, G., et al. 2014, ApJL, 793, L22

Foley, R. J., McCully, C., Jha, S. W., Bildsten, L., Fong, W.-f., Narayan, G., Rest, A., \& Stritzinger, M. D. 2014, ApJ, 792, 29

Foley, R. J., et al. 2011, ApJ, 732, 32

Foley, R. J., et al. 2013, ApJ, 767, 57

Fraser, M. et al. 2010, ApJL, 714, L280

Fraser, M. et al. 2013, MNRAS, 433, 1312

Fraser, M. et al. 2014, MNRAS, 439, L56

Fruchter, A. S., et al. 2006, Nature, 441, 463

Galama, T. J., et al. 1998, Nature, 395, 670

Galbany, L., et al. 2014, A\&A, 572, A38

Gal-Yam, A., \& Leonard, D. C. 2009, Nature, 458, 865

Georgy, C., Meynet, G., Walder, R., Folini, D., \& Maeder, A. 2009, A\&A, 502, 611

Gogarten, S. M., Dalcanton, J. J., Murphy, J. W., Williams, B. F., Gilbert, K., \& Dolphin, A. 2009a, ApJ, 703, 300

Gogarten, S. M., et al. 2009b, ApJ, 691, 115

Graham, M. L., et al. 2014, ApJ, 787, 163

Gutiérrez, C. P., et al. 2014, ApJL, 786, L15

Habergham, S. M., Anderson, J. P., \& James, P. A. 2010, ApJ, 717, 342

Habergham, S. M., Anderson, J. P., James, P. A., \& Lyman, J. D. 2014, MNRAS, 441, 2230

Habergham, S. M., James, P. A., \& Anderson, J. P. 2012, MNRAS, 424, 2841

Hakobyan, A. A., Mamon, G. A., Petrosian, A. R., Kunth, D., \& Turatto, M. 2009, A\&A, 508, 1259

Hakobyan, A. A., et al. 2014, MNRAS, 444, 2428

Heger, A., Fryer, C. L., Woosley, S. E., Langer, N., \& Hartmann, D. H. 2003, ApJ, 591, 288

Henry, R. B. C., \& Worthey, G. 1999, PASP, 111, 919

Hibbard, J. E., \& van Gorkom, J. H. 1996, AJ, 111, 655

Hjorth, J., et al. 2003, Nature, 423, 847

Huang, Y.-L. 1987, PASP, 99, 461

Ibeling, D., \& Heger, A. 2013, ApJL, 765, L43

Inserra, C., et al. 2013, A\&A, 555, A142

James, P. A., \& Anderson, J. P. 2006, A\&A, 453, 57

Jennings, Z. G., et al. 2014, ApJ, 795, 170

Johnson, H. M., \& MacLeod, J. M. 1963, PASP, 75, 123

Joseph, R. D., \& Wright, G. S. 1985, MNRAS, 214, 87

Kangas, T., Mattila, S., Kankare, E., Kotilainen, J. K., Väisänen, P., Greimel, R., \& Takalo, A. 2013, MNRAS, 436, 3464 
Kasliwal, M. M., et al. 2012, ApJ, 755, 161

Kawabata, K. S., et al. 2010, Nature, 465, 326

Kelly, P. L., Filippenko, A. V., Burke, D. L., Hicken, M., Ganeshalingam, M., \& Zheng, W. 2014, Science, 347, 1459

Kelly, P. L., \& Kirshner, R. P. 2012, ApJ, 759, 107

Kelly, P. L., Kirshner, R. P., \& Pahre, M. 2008, ApJ, 687, 1201

Kennicutt, Jr., R. C. 1998, ARA\&A, 36, 189

Kewley, L. J., \& Ellison, S. L. 2008, ApJ, 681, 1183

Kewley, L. J., Geller, M. J., \& Barton, E. J. 2006, AJ, 131, 2004

Kewley, L. J., Rupke, D., Jabran Zahid, H., Geller, M. J., \& Barton, E. J. 2010, ApJL, 721, L48

Kiewe, M., et al. 2012, ApJ, 744, 10

Kleiser, I. K. W., et al. 2011, MNRAS, 415, 372

Klessen, R. S., Spaans, M., \& Jappsen, A. 2007, MNRAS, 374, L29

Kobulnicky, H. A., \& Fryer, C. L. 2007, ApJ, 670, 747

Kochanek, C. S., Szczygieł, D. M., \& Stanek, K. Z. 2012, ApJ, 758, 142

Kudritzki, R.-P., \& Puls, J. 2000, ARA\&A, 38, 613

Kuncarayakti, H., et al. 2013a, AJ, 146, 30

Kuncarayakti, H., et al. 2013b, AJ, 146, 31

Kuncarayakti, H., et al. 2014, arXiv e-prints: 1410.8739

Lada, C. J., \& Lada, E. A. 2003, ARA\&A, 41, 57

Larson, R. B., \& Tinsley, B. M. 1978, ApJ, 219, 46

Law, D. R., \& MaNGA Team 2014, in American Astronomical Society Meeting Abstracts, Vol. 223 (Washington, DC), 254.31

Leloudas, G., Sollerman, J., Levan, A. J., Fynbo, J. P. U., Malesani, D., \& Maund, J. R. 2010, A\&A, 518, A29

Leloudas, G., et al. 2011, A\&A, 530, A95

Leloudas, G., et al. 2015, MNRAS, 449, 917

Li, W., et al. 2011, MNRAS, 412, 1441

Lunnan, R., et al. 2014a, ApJ, 787, 138

Lunnan, R., et al. 2014b, arXiv e-prints: 1411.1060

Lyman, J., Bersier, D., James, P., Mazzali, P., Eldridge, J., Fraser, M., \& Pian, E. 2014a, arXiv e-prints: 1406.3667

Lyman, J. D., James, P. A., Perets, H. B., Anderson, J. P., Gal-Yam, A., Mazzali, P., \& Percival, S. M. 2013, MNRAS, 434, 527

Lyman, J. D., Levan, A. J., Church, R. P., Davies, M. B., \& Tanvir, N. R. 2014b, MNRAS, 444, 2157

Margutti, R., et al. 2014, ApJ, 780, 21

Mauerhan, J., et al. 2014, MNRAS, 442, 1166

Mauerhan, J. C., et al. 2013, MNRAS, 430, 1801

Maund, J. R., \& Smartt, S. J. 2009, Science, 324, 486

Maund, J. R., Smartt, S. J., Kudritzki, R. P., Podsiadlowski, P., \& Gilmore, G. F. 2004, Nature, 427, 129

Maund, J. R., et al. 2006, MNRAS, 369, 390

Maund, J. R., et al. 2011, ApJL, 739, L37

Maund, J. R., et al. 2013, MNRAS, 431, L102

Maza, J., \& van den Bergh, S. 1976, ApJ, 204, 519

McCully, C., Jha, S. W., Foley, R. J., Bildsten, L., Fong, W.-F., Kirshner, R. P., Marion, G. H., Riess, A. G., \& Stritzinger, M. D. 2014, Nature, 512, 54

McMillan, R. J., \& Ciardullo, R. 1996, ApJ, 473, 707

Mihos, J. C., \& Hernquist, L. 1996, ApJ, 464, 641

Milisavljevic, D., et al. 2013, ApJ, 767, 71

Minkowski, R. 1941, PASP, 53, 224

Modjaz, M. 2011, AN, 332, 434

Modjaz, M., Kewley, L., Bloom, J. S., Filippenko, A. V., Perley, D., \& Silverman, J. M. 2011, ApJL, 731, L4

Modjaz, M., et al. 2008, AJ, 135, 1136

Modjaz, M., et al. 2014, AJ, 147, 99
Mokiem, M. R., et al. 2007, A\&A, 473, 603

Murphy, J. W., Jennings, Z. G., Williams, B., Dalcanton, J. J., \& Dolphin, A. E. 2011, ApJL, 742, L4

Neill, J. D., et al. 2011, ApJ, 727, 15

Pan, Y.-C., et al. 2015, MNRAS, 446, 354

Pastorello, A., et al. 2005, MNRAS, 360, 950

Pastorello, A., et al. 2012, A\&A, 537, A141

Pastorello, A., et al. 2013, ApJ, 767, 1

Perets, H. B., et al. 2010, Nature, 465, 322

Perets, H. B., et al. 2011, ApJL, 728, L36

Pettini, M., \& Pagel, B. E. J. 2004, MNRAS, 348, L59

Phillips, M. M., et al. 2013, ApJ, 779, 38

Podsiadlowski, P., Joss, P. C., \& Hsu, J. J. L. 1992, ApJ, 391, 246

Portegies Zwart, S. F., McMillan, S. L. W., \& Gieles, M. 2010, ARA\&A, 48, 431

Prantzos, N., \& Boissier, S. 2003, A\&A, 406, 259

Prieto, J. L., Brimacombe, J., Drake, A. J., \& Howerton, S. 2013, ApJL, 763, L27

Prieto, J. L., Stanek, K. Z., \& Beacom, J. F. 2008a, ApJ, 673, 999

Prieto, J. L., et al. 2008b, ApJL, 681, L9

Prieto, J. L., et al. 2012, ApJ, 745, 70

Puls, J., et al. 1996, A\&A, 305, 171

Rampazzo, R., Plana, H., Amram, P., Bagarotto, S., Boulesteix, J., \& Rosado, M. 2005, MNRAS, 356, 1177

Raskin, C., Scannapieco, E., Rhoads, J., \& Della Valle, M., 2008, ApJ, 689, 358

Raskin, C., Scannapieco, E., Rhoads, J., \& Della Valle, M., 2009, ApJ, 707, 74

Rau, A., et al. 2009, PASP, 121, 1334

Reaves, G. 1953, PASP, 65, 242

Rigault, M., et al. 2013, A\&A, 560, A66

Rigault, M., et al. 2015, ApJ, 802, 20

Sana, H., et al. 2012, Science, 337, 444

Sana, H., et al. 2013, A\&A, 550, A107

Sánchez, S. F., et al. 2012, A\&A, 538, A8

Sánchez, S. F., et al. 2014, A\&A, 563, A49

Sánchez, S. F., et al. 2015, A\&A, 573, A105

Sanders, N. E., et al. 2012, ApJ, 758, 132

Sanders, N. E., et al. 2015, ApJ, 799, 208

Schlegel, E. M. 1990, MNRAS, 244, 269

Simón-Díaz, S., \& Stasińska, G. 2011, A\&A, 526, A48

Smartt, S. J. 2009, ARA\&A, 47, 63

Smartt, S. J., Eldridge, J. J., Crockett, R. M., \& Maund, J. R. 2009, MNRAS, 395, 1409

Smith, N. 2008, in Proc. IAU Symp., Vol. 250, Massive Stars as Cosmic Engines, ed. S. Kwok, \& S. A. Sanford (Cambridge: Cambridge University Press), 193

Smith, N. 2013, MNRAS, 434, 102

Smith, N., Li, W., Silverman, J. M., Ganeshalingam, M., \& Filippenko, A. V. 2011, MNRAS, 415, 773

Smith, N., Mauerhan, J. C., \& Prieto, J. L. 2014, MNRAS, 438, 1191

Smith, N., \& Tombleson, R. 2015, MNRAS, 447, 598

Smith, N., et al. 2010, AJ, 139, 1451

Stanishev, V., Rodrigues, M., Mourão, A., \& Flores, H. 2012, A\&A, 545, A58

Stoll, R., Prieto, J. L., Stanek, K. Z., \& Pogge, R. W. 2013, ApJ, 773, 12

Taddia, F., et al. 2012, A\&A, 537, A140

Taddia, F., et al. 2013a, A\&A, 558, A143 
Taddia, F., et al. 2013b, A\&A, 555, A10

Thöne, C. C., et al. 2009, ApJ, 698, 1307

Thöne, C. C., et al. 2014, arXiv e-prints: 1411.1104

Tomasella, L., et al. 2013, MNRAS, 434, 1636

Tsvetkov, D. Y., Pavlyuk, N. N., \& Bartunov, O. S. 2004, AstL, 30, 729

Valenti, S., et al. 2014, MNRAS, 437, 1519

van den Bergh, S. 1997, AJ, 113, 197

van den Bergh, S., Li, W., \& Filippenko, A. V. 2005, PASP, 117, 773

van Dyk, S. D. 1992, AJ, 103, 1788

van Dyk, S. D., Hamuy, M., \& Filippenko, A. V. 1996, AJ, 111, 2017 van Dyk, S. D., Peng, C. Y., King, J. Y., Filippenko, A. V., Treffers, R. R., Li, W., \& Richmond, M. W. 2000, PASP, 112, 1532

Van Dyk, S. D., et al. 2011, ApJL, 741, L28

Van Dyk, S. D., et al. 2012, AJ, 143, 19

Wang, L., Höflich, P., \& Wheeler, J. C. 1997, ApJL, 483, L29

Wang, X., Wang, L., Filippenko, A. V., Zhang, T., \& Zhao, X. 2013, Science, 340, 170

Williams, B. F., et al. 2014, ApJ, 791, 105

Woosley, S. E., \& Bloom, J. S. 2006, ARA\&A, 44, 507

Wright, N. J., Parker, R. J., Goodwin, S. P., \& Drake, J. J. 2014, MNRAS, 438, 639

Yuan, F., Kobayashi, C., Schmidt, B. P., Podsiadlowski, P., Sim, S. A., \& Scalzo, R. A. 2013, MNRAS, 432, 1680 